\title{
Hilft viel viel? Der Einfluss von Studienstrukturen auf das Professionswissen angehender Physiklehrkräfte
}

\author{
Dustin Schiering $(\mathbb{D}) \cdot$ Stefan Sorge $(\mathbb{D}) \cdot$ Knut Neumann $(\mathbb{D}$
}

Eingegangen: 23. Januar 2020 / Überarbeitet: 7. September 2020 / Angenommen: 16. Februar 2021 /

Online publiziert: 4. März 2021

(C) Der/die Autor(en) 2021

Zusammenfassung Universitäre Lerngelegenheiten sind zentral für den Erwerb des Fachwissens (FW) und fachdidaktischen Wissens (FDW) angehender Lehrkräfte. Die Unterschiede in Umfang und Inhalt der Lerngelegenheiten zwischen den Hochschulen sind jedoch groß. Am Beispiel des Physik-Lehramtsstudiums werden in dieser Studie verschiedene Studienstrukturen und ihr Einfluss auf das FW und FDW angehender Lehrkräfte analysiert. Hierzu wurden Curricula von 20 deutschen Hochschulen für das Lehramt verglichen und Inhalt sowie Umfang ihrer fachund fachdidaktischen Anteile in den ersten sechs Semestern identifiziert. Mit Blick auf die fachliche Ausbildung angehender Lehrkräfte zeigte sich, dass sie den Umfang der fachdidaktischen Ausbildung um etwa das Fünffache übersteigt. Zudem konnten vier Cluster identifiziert werden, die als prototypische Curricula mit geringem bzw. umfangreichen Fach- und Fachdidaktikanteil interpretiert werden können. Cluster mit umfangreichen Fachanteilen wirken sich dabei signifikant besser auf das FW aus. Zudem ist der Zusammenhang von FW und FDW abhängig von der Studienstruktur, so hängt das FDW von Probanden aus Studiengängen mit geringen Fachdidaktikanteilen stärker vom FW ab.

Schlüsselwörter Fachdidaktisches Wissen · Fachwissen · Lehramtsausbildung · Physik · Studienstrukturen

\footnotetext{
D. Schiering $(\varangle) \cdot$ Dr. S. Sorge $\cdot$ Prof. Dr. K. Neumann Abteilung Didaktik der Physik, IPN - Leibniz-Institut für die Pädagogik der Naturwissenschaften und Mathematik, Olshausenstraße 62, 24118 Kiel, Deutschland

E-Mail: schiering@leibniz-ipn.de

Dr. S. Sorge

E-Mail: sorge@ @eibniz-ipn.de

Prof. Dr. K. Neumann

E-Mail: neumann@leibniz-ipn.de
} 


\title{
The more, the better? The influence of learning opportunities in physics teacher training programs
}

\begin{abstract}
Learning opportunities in teacher training programs are crucial for the development of pre-service teachers' content knowledge (CK) and pedagogical content knowledge (PCK). However, German teacher training programs (TTP) differ in their structure, particularly in their amount and content of learning opportunities. The present study characterizes different TTP and analyzes their influence on preservice physics teachers' CK and PCK. Therefore, curricula of 20 German TTP were compared and the amount and content of their learning opportunities during the first three years of enrollment were examined. Findings show that the amount of physics-specific learning opportunities is five times greater than the amount of learning opportunities related to physics education. Furthermore, four clusters can be identified which can be interpreted as TTP with more/less learning opportunities for physics and physics education. Clusters with more learning opportunities for physics have a positive influence on pre-service teachers' CK. For clusters with less learning opportunities for physics education pre-service teachers rely more on their CK for their PCK.
\end{abstract}

Keywords Content knowledge · Pedagogical content knowledge · Physics · Teacher education $\cdot$ Teacher training program

\section{Einleitung}

In den letzten Jahrzehnten hat die Bildungsforschung wiederholt gezeigt, dass die Lehrkraft ein entscheidender Faktor für erfolgreichen Unterricht ist. Dabei ist das Professionswissen der Lehrkraft, insbesondere die fachspezifischen Wissensbereiche Fachwissen (FW) und fachdidaktisches Wissen (FDW), entscheidend für den Lernerfolg der Schülerinnen und Schüler (Baumert und Kunter 2011). Die bisherige Forschung legt nahe, dass dieses Wissen maßgeblich im universitären Teil der Lehramtsausbildung erworben wird (z. B. Brunner et al. 2006; Kleickmann et al. 2013). Zur Gestaltung der universitären Lehramtsausbildung verabschiedete die Kultusministerkonferenz (KMK) in den letzten Jahrzehnten diverse Beschlüsse, die die inhaltlichen Anforderungen des Lehramtsstudiums mit dem Ziel konkretisieren, das Lehramtsstudium über die Bundesländer vergleichbarer zu gestalten und Aussagen über die Qualifikation der Lehrkräfte treffen zu können (KMK 2005).

Die Standards der Lehrkräftebildung auf der einen Seite und die Länder- und Hochschulautonomie auf der anderen Seite mündeten jedoch darin, dass sich verschiedene Lehramtsprogramme bezüglich Umfang und Inhalten der Lehrveranstaltungen stark unterscheiden (vgl. z. B. Keller 2010) und diese Unterschiede mit dem Professionswissen der angehenden Lehrkräfte korrelieren. So konnten zum Beispiel Großschedl et al. (2015) feststellen, dass das FW und FDW von gewählten Lehramtstyp (gymnasiales Lehramt versus nicht-gymnasiales Lehramt) abhängig ist. Darüber hinaus zeigten Studien zu pädagogischen Lerngelegenheiten, dass nicht nur der Lehramtstyp, sondern auch Inhalt und Umfang der eigentlichen Lerngelegenheiten 
mit dem pädagogischen Wissen angehender Lehrkräfte zusammenhängen (vgl. z.B. Kunina-Habenicht et al. 2013; Tachtsoglou und König 2017; Watson et al. 2018). Bisher fehlen allerdings Untersuchungen zum fachspezifischen Professionswissen, die es ermöglichen festzustellen, wie sich ,welches Element innerhalb welcher Lehrerbildung auf welche Teile der Lehrerkompetenz [auswirkt]“ (Terhart 2012, S. 14) insbesondere in der Ausbildung von Physiklehrkräften.

Die vorliegende Studie beschäftigt sich aus diesem Grund mit den in Deutschland vorliegenden Studienstrukturen und deren Einfluss auf das FW und FDW angehender Physiklehrkräfte. Hierzu wurden zunächst die Curricula von 20 Hochschulen systematisch analysiert und anhand ihrer Anteile in Fach- und Fachdidaktik klassifiziert. Anschließend wurden Daten von $N=107$ Physik-Lehramtsstudierenden genutzt, um Zusammenhänge zwischen den Studienstrukturen und dem FW und FDW der angehenden Physiklehrkräfte zu charakterisieren.

\section{Theoretischer Hintergrund}

\subsection{Die universitäre Lehramtsausbildung in Deutschland}

\subsubsection{Studienmodelle für das allgemeinbildende Lehramt}

Die Bologna-Reform der Hochschulausbildung 1999 hatte zum Ziel, das Studium künftig so zu organisieren, dass eine höhere Vergleichbarkeit der Studiengänge und Flexibilität der Studierenden über die nationalen Grenzen hinaus gewährleistet sind (Bauer et al. 2012). Vor diesem Hintergrund wurden traditionelle Studienstrukturen in Deutschland, darunter auch das Staatsexamen in Lehramtsstudiengängen, größtenteils durch eine modularisierte und gestufte Studienstruktur, das Bachelor-MasterModell, ersetzt (Eurydice 2010). Die Umstellung auf das Bachelor-Master-Modell stellt jedoch das Lehramtsstudium vor eine besondere Herausforderung (z. B. Bauer et al. 2011). Einerseits soll im Bachelor-Master-Modell der Bachelor als polyvalenter Studiengang angeboten werden, was den Bachelor als ersten sogenannten berufsqualifizierenden Abschluss etabliert (Keuffer 2010). Somit qualifiziert der polyvalente Bachelor neben dem Lehramt auch für andere Berufsfelder und Masterstudiengänge, was vorsieht, dass Studierende erst nach Erwerb des Bachelorabschlusses die Entscheidung treffen, das Lehramtsstudium aufzunehmen (Terhart 2008). Dies würde bedeuten, dass Studiengänge im Bachelor vermehrt Fachwissenschaften lehren und die Ausbildung in den Erziehungswissenschaften und Fachdidaktik hauptsächlich im Master stattfindet (sequenzielles Modell) (vgl. Terhart 2008). Andererseits aber wird von der KMK (2005) die thematische Trennung von Bachelor und Master verringert, indem sie Bildungswissenschaften sowie schulpraktische Studien als festen Teil des Bachelor-Studiums festschreiben. Folglich findet bereits im Bachelor eine lehramtsspezifische Ausbildung statt (integratives Modell), wodurch sich Studierende aufgrund von Formalitäten tendenziell sehr früh (im Bachelor) auf das Lehramtsstudium festlegen müssen (Bauer et al. 2011). Dies legt die Vermutung nahe, dass der Bachelor de facto nur als Funktion einer Zwischenprüfung (analog 
zur Zwischenprüfung im Studienmodell Staatsexamen) besteht (Terhart 2012) und „inneruniversitär keine Funktion“ (Weiler 2007, S. 26) trägt.

Der polyvalente Bachelor auf der einen Seite und die Vorgaben der KMK (2005) zu lehramtsspezifischen Inhalten im Bachelor auf der anderen führten zu ganz unterschiedlichen Studienstrukturen (Bauer et al. 2012). So kommt beispielsweise Keuffer (2010) zu dem Schluss, dass die Reformen der Studienstrukturen tatsächlich eine stärkere Heterogenität im Lehramtsstudium hervorbrachten als zuvor; Keller (2010) spricht von einem ,,bunte[n] Flickenteppich unterschiedlicher Varianten der Einführung oder auch Nichteinführung von Bachelor- und Masterstudiengängen in der Lehrerbildung“ (Keller 2010, S. 102). Vor diesem Hintergrund attestieren Bauer et al. (2012) der aktuellen Studiensituation für allgemeinbildende Lehrämter in Deutschland einen hohe Heterogenität. Welche Auswirkungen diese Heterogenität jedoch auf die Struktur des Lehramtsstudiums speziell im Fach Physik hat, ist bislang nicht klar. Einen ersten Hinweis hierfür können Standards, Curricula und Richtlinien zur Ausbildung von Physiklehrkräften geben.

\subsubsection{Lehramtsstudium Physik: Standards und Richtlinien}

Spricht man über Studienstrukturen, so müssen die universitären Curricula auf drei Ebenen unterschieden werden: Intendiertes, implementiertes sowie erreichtes Curriculum (Hascher 2011). Tachtsoglou und König (2017) führen hierzu aus, dass das intendierte Curriculum Inhalte und Anforderungen in Form von Prüfungs- oder Studienordnungen verbindlich formalisiert. Das implementierte Curriculum beschreibt die tatsächliche Umsetzung des intendierten Curriculums. Das erreichte Curriculum fokussiert schließlich das Können und Wissen der Lernenden und ist somit als Resultat der beiden anderen Curricula zu verstehen (Hascher 2011). Während zur Untersuchung des erreichten Curriculums ein Instrument zur Erfassung der Lernziele (hier der professionellen Kompetenzen angehender Physiklehrkräfte) nötig ist und das implementierte Curriculum mindestens eine Befragung der Lehrenden erfordert, sind intendierte Curricula oft öffentlich zugänglich und dienen als rechtlicher Rahmen von und für Hochschulen sowie Dozierende.

Die Grundlage intendierter Curricula der Lehrkräftebildung bildet der Beschluss der KMK (2019). Darin werden die zu erreichenden fachspezifischen Kompetenzen der angehenden Lehrkräfte sowie Studieninhalte für Fachwissenschaft und Fachdidaktik definiert. Damit Lehrkräfte am Ende ihrer Ausbildung entsprechende fachspezifische Kompetenzen erreichen, nennt die KMK (2019) Studieninhalte, die als inhaltliche Vorgabe des Physik-Lehramtsstudiums dienen. Untergliedert sind diese Studieninhalte in sechs Themenbereiche und dazugehörige Inhalte. So finden sich unter dem Themenbereich „Experimentalphysik“ die Inhalte Mechanik, Thermodynamik, Elektrodynamik und Optik, Atom- und Quantenphysik sowie Festkörper-, Kern- und Elementarteilchenphysik, Kosmologie. Der Themenbereich „Theoretische Physik" umfasst die Inhalte Theoretische Mechanik, Thermodynamik, Elektrodynamik und Quantentheorie. Unter dem Themenbereich „Physikdidaktik“ führt die KMK (2019) die Inhalte Fachdidaktische Positionen und Konzeptionen, Motivation und Interesse, Lernprozesse, Diagnose von Lernschwierigkeiten, Planung und Analyse von Physikunterricht, Aufgaben, Experimente und Medien, Umgang 
mit Heterogenität im Physikunterricht sowie Fachdidaktische Forschung auf. Weitere Themenbereiche sind physikalische Praktika, Mathematik für Physik und angewandte Physik, auf die hier nicht im Detail eingegangen wird. Zu welchem Zeitpunkt des Lehramtsstudiums oder in welchem Umfang die Inhalte behandelt werden sollen, ist nicht festgelegt, da ,die Zuordnung von Inhalten zu Veranstaltungen [...] in Studienplänen zu treffen [ist]“ (KMK 2019, S. 5), somit also der Länder- und Hochschulautonomie unterliegt.

Die Deutsche Physikalische Gesellschaft [DPG] (2014) veröffentlichte ihrerseits Empfehlungen zur Strukturierung des Physik-Lehramtsstudiums. Die darin beschriebenen Kompetenzen angehender Physiklehrkräfte explizieren die der KMK (2019), gehen teilweise auch darüber hinaus. So empfiehlt die DPG (2014), beispielsweise den Studieninhalt Natur der Naturwissenschaften („Nature of Science“) explizit als eigenen Inhalt in die fachdidaktischen Ausbildung aufzunehmen. Zudem wird ein detaillierter Vorschlag für den Studienverlauf hinsichtlich der fachlichen wie auch fachdidaktischen Inhalte vorgegeben. Inwieweit jedoch dieser und andere exemplarische Vorschläge an den einzelnen Hochschulen tatsächlich in die Modulpläne aufgenommen wurden, ist fraglich, gerade weil Hochschulen im „Spannungsverhältnis zwischen Hochschulautonomie und föderalen Bildungsentscheidungen der Kultus- und Wissenschaftsministerien zu sehen [sind]“ (Bauer et al. 2012, S. 105). Es ist demnach allein schon auf Grund der bestehenden Studienmodelle (Staatsexamen vs. Bachelor-Master-Modell) zu erwarten, dass das intendierte Curriculum des Physik-Lehramtsstudiums eine hohe Heterogenität aufweist. Zudem ist anzunehmen, dass sich eben diese Heterogenität in den erreichten Curricula, also dem Erwerb von Fachwissen und fachdidaktischen Wissen angehender Physiklehrkräfte, niederschlagen wird (vgl. Riese und Reinhold 2010).

\subsection{Das Fachwissen und fachdidaktische Wissen als Teil des Professionswissens}

Die einflussreichste Modellierung des Professionswissens geht auf Shulman (1987) zurück, der sieben Aspekte des Professionswissens formulierte, von denen das Fachwissen (FW), fachdidaktische Wissen (FDW) und das pädagogisch-psychologische Wissen die ,allgemein akzeptierten Kernkategorien des Professionswissens von Lehrkräften darstellen“ (Krauss 2011, S. 181). Forscherinnen und Forscher folgten in den letzten Jahrzehnten dieser Modellierung und beschrieben die fachbezogenen Wissenskategorien - das Fachwissen (FW) sowie fachdidaktische Wissen (FDW) genauer (Kleickmann et al. 2013).

Das FW umfasst ein tiefes Verständnis der Struktur und Inhalte der Domäne (z. B. Grossman 1990; Krauss et al. 2008), sodass Lehrkräfte befähigt werden, Zusammenhänge in der Domäne zu erkennen. Folglich wird das FW üblicherweise in zwei Dimensionen unterschieden: Art des Wissens und Inhalt (Tepner et al. 2012). Die möglichen Inhaltsdimensionen des FW (also den zentralen Themen der Physik) verhalten sich weitestgehend konsensuell und lassen sich in den von der KMK (2019) definierten Studieninhalten identifizieren (Sorge et al. 2019). Die Art des Wissens wird dabei in Anlehnung an Shavelson et al. (2005) in deklaratives, prozedurales und strategisches bzw. konditionales Wissen unterschieden. 
Die Modellierungen des FDW sind im Vergleich zu denen des FW weniger übereinstimmend (für einen Überblick Gramzow et al. 2013). Daraus folgt, dass die Operationalisierungen des FDW nur schwer vergleichbar sind. Dennoch stützen sich alle Forschungsarbeiten zum FDW zunächst auf Shulmans (1986) Konzeptualisierung als Wissen, mit dessen Hilfe Lehrkräfte Fachinhalte aufbereiten können, um sie den Schülerinnen und Schülern verständlich zu machen (Shulman 1986). Eine innere Systematik erlangt das FDW durch sogenannte Facetten. Hierzu existieren bereits detaillierte Übersichtsarbeiten zu möglichen Facetten (z. B. Kind 2009; Kirschner 2013). Weitestgehend wird das Modell von Magnusson et al. (1999) als Konsens für die Beschreibung der Facetten des FDW angesehen (Kind 2009; Park und Oliver 2008). Darin postulieren Magnusson et al. (1999) eine Unterteilung des FDW in die fünf Facetten Wissen über Curriculum, Vorstellungen der Schülerinnen und Schüler, Assessment, Instruktionsstrategien sowie Orientierungen gegenüber naturwissenschaftlichem Unterricht. Die Orientierungen gegenüber dem naturwissenschaftlichen Unterricht als Wissensfacette zu verstehen, ist jedoch als problematisch zu sehen (z. B. Schiering et al. 2019). Darüber hinaus wird dem FDW in Modellen wie dem Consensus Model (Gess-Newsome 2015) oder dem Refined Consensus Model (Carlson und Daehler 2019) eine handelnde Komponente ergänzt, indem das FDW sowohl das Wissen über Unterrichten als auch die eigentliche Handlung im Unterricht umfasst (im Sinne einer professioneller Handlungskompetenz nach Baumert und Kunter 2006). Dabei stellt das Wissen eine wichtige Voraussetzung für das Handeln im Unterricht dar (z.B. auch Blömeke et al. 2015), weshalb in der universitären Ausbildung der Erwerb von fachdidaktischem Wissen fokussiert wird.

Mittlerweile zeigt eine Reihe von Studien, dass die Trennung von FW und FDW empirisch möglich ist (z. B. Kirschner et al. 2016; Kleickmann et al. 2013; Sorge et al. 2019). Somit kann angenommen werden, dass es sich beim FW und FDW um zwei sowohl theoretisch als auch empirisch trennbare (aber hoch korrelierte) Bereiche des Professionswissens von Lehrkräften handelt (vgl. Neumann et al. 2018).

\subsection{Die Entwicklung von FW und FDW im Rahmen der universitären Ausbildung}

Die physikdidaktische Forschung zur Entwicklung des FW und FDW angehender Lehrkräfte kann bereits auf eine Vielzahl von Projekten und Arbeiten zurückblicken. Dabei konnten sich die Arbeiten im Bereich der Physik zunächst auf Erkenntnisse der Mathematik beziehen (z. B. Brunner et al. 2006; Schmidt et al. 2007) und schließlich eigenständige Ergebnisse präsentieren. Aufbauend auf den ersten Modellierungen der professionellen Kompetenz von Physiklehrkräften (z.B. Riese 2009) widmete man sich in Projekten wie ProwiN (Borowski et al. 2010) oder QuiP (Fischer et al. 2014) der Frage, welchen Einfluss das Professionswissen auf den Unterricht und das Lernen der Schülerinnen und Schüler hat. In weiteren Projekten rückten dann speziell angehende Physiklehrkräfte in den Fokus, wobei ihr Wissen (z. B. für KiL: Kröger 2019; für ProfiLe-P: Riese et al. 2015; für FALKO-P: Schödl 2017), ihre naturwissenschaftlichen Arbeitsweisen (z. B. für Ko-WADiS: Straube 2016) und der Zusammenhang zwischen Wissen und Handeln (z. B. für Phi-Actio: Korneck et al. 2017; für ProfiLe-P+: Vogelsang et al. 2020) genauer untersucht wurden. 
Auf Grundlage dieser Forschung konnten in den letzten Jahren die Erkenntnisse zur Entwicklung des FW und FDW angehender Physiklehrkräfte weiter konkretisiert werden. So stehen den angehenden Physiklehrkräften während der universitären Ausbildung verschiedene Lerngelegenheiten zur Verfügung, wobei formale Lerngelegenheiten wie Vorlesungen und Seminare eine zentrale Rolle in der Förderung von FW und FDW einnehmen (Sorge et al. 2019). Diverse Studien mit angehenden Physiklehrkräften konnten zeigen, dass fortgeschrittene Studierende über ein höheres FW und FDW verfügen als Studienanfänger (z. B. Reinhold und Riese 2012; Riese et al. 2017; Sorge et al. 2019; siehe auch Chan und Kind 2019; Großschedl et al. 2015; Kleickmann et al. 2014). Dieser Unterschied im Wissen spricht für eine generelle Wirksamkeit des Lehramtsstudiums (Anders und Kleickmann 2011; Schödl 2017). Beide Wissensbereiche entwickeln sich jedoch nicht getrennt voneinander. So gilt das FW als notwendige, aber nicht hinreichende Bedingung für das FDW (z. B. Chan und Kind 2019; Reinhold und Riese 2010). Diverse Studien zeigen entsprechend einen signifikanten Zusammenhang zwischen dem FW und FDW angehender und praktizierender Physiklehrkräfte (z. B. Borowski und Liepertz 2018; Kirschner et al. 2017; Schödl 2017; Sorge et al. 2019). Neben diesen Ergebnissen zum Zusammenhang von FW und FDW zeigen bisherige Arbeiten auch, dass deren Höhe vom gewählten Lehramtstyp (gymnasiales Lehramt vs. Haupt- und Realschullehramt) abhängt (z. B. Reinhold und Riese 2012). So zeigte sich zum Beispiel, dass angehende Gymnasiallehrkräfte ein höheres FW aufweisen als angehende Hauptund Realschullehrkräften (Physik: Kirschner et al. 2017; Schödl 2017; Mathematik: Kleickmann et al. 2013; Biologie: Großschedl et al. 2015). Für das FDW zeigte sich jedoch in Untersuchungen von Reinhold und Riese (2012) sowie Sorge et al. (2019), dass angehende Gymnasiallehrkräfte in Physik nicht zwangsläufig ein höheres FDW besitzen als angehende Haupt- und Realschullehrkräfte in Physik.

Zusammenfassend zeigen die bisherigen Arbeiten, dass die universitäre Ausbildung angehender Physiklehrkräfte signifikante Einflüsse sowohl auf das FW als auch auf das FDW hat (z. B. Sorge et al. 2019). Darüber hinaus scheint ein fundiertes FW Grundlage für ein adäquates FDW zu sein (z. B. Reinhold und Riese 2010). Insgesamt liefern bisherige Untersuchungen somit wichtige Ergebnisse, jedoch scheinen Merkmale wie Lehramts- oder Hochschultyp nicht differenziert genug zu sein, um detaillierte Rückschlüsse auf das FW und FDW angehender Physiklehrkräfte zuzulassen.

\section{Fragestellungen}

Die universitäre Lehramtsausbildung stellt einen entscheidenden Faktor für die Entwicklung des FW und FDW angehender Lehrkräfte dar (z. B. Kleickmann und Anders 2011). Offen ist aber, wie sich verschiedene Studienstrukturen auf das FW und FDW auswirken. Zwar liegen vereinzelt Studien vor, die die Studienzeit mit dem Wissenserwerb verknüpfen, es fehlen jedoch detaillierte Untersuchungen zu verschiedenen Arten universitärer Lehramtsausbildungen angehender Physiklehrkräfte und inwieweit sich die Vorgaben der KMK (2019) tatsächlich in den Modulplänen des Lehramtsstudiums wiederfinden lassen. Somit ist es nötig, eine systematische 
Untersuchung von intendierten Curricula der Hochschulen, wie auch deren Manifestation im FW und FDW angehender Physiklehrkräfte durchzuführen. Demnach ergeben sich für diese Studien folgende Forschungsfragen:

1. FF Welche fachlichen und fachdidaktischen Themen und Inhalte kennzeichnen das Physik-Lehramtsstudium in Deutschland?

2. FF Wie lassen sich die verschiedenen Physik-Lehramtsstudiengänge hinsichtlich ihrer Fach- und Fachdidaktikanteile klassifizieren?

3. FF Welchen Einfluss haben unterschiedliche Studienstrukturen auf das Fachwissen und fachdidaktische Wissen angehender Physiklehrkräfte?

Das Spannungsverhältnis zwischen Polyvalenzforderung und Vorgaben der KMK (2005) mündete in einer Vielzahl von verschiedenen Studienstrukturen. Bislang ist es weitestgehend unklar, ob die erste Hälfte des Physik-Lehramtsstudiums hauptsächlich Fachinhalte beinhaltet oder bereits lehramtsspezifische Inhalte fördert (sequenzielles vs. integratives Modell). Es stellt sich somit die Frage, wie sich gerade die erste Hälfte des Physik-Lehramtsstudiums auf das FW und FDW angehender Lehrkräfte auswirkt. Zudem hat die in Deutschland herrschende Heterogenität in den Studienstrukturen auch Auswirkungen auf die Regelstudienzeit des Physik-Lehramtsstudiums, ,sodass die Ausbildungsdauer im bundesweiten Vergleich nach wie vor variiert" (Radhoff und Ruberg 2016, S. 47), mindestens jedoch sechs Semester beträgt. Um also einerseits die erste Hälfte des Physik-Lehramtsstudiums genauer beschreiben zu können und andererseits eine möglichst hohe Anzahl an verschiedenen Studienstrukturen miteinander zu vergleichen, fokussiert diese Studie die ersten sechs Semester des Physik-Lehramtsstudiums.

\section{Methoden}

Zur Beantwortung der oben formulierten Forschungsfragen wurden zwei Methoden miteinander kombiniert. Zunächst sollen die vorherrschenden Studienstrukturen für das Physik-Lehramtsstudium in Deutschland identifiziert werden, anschließend soll das FW und FDW von angehenden Physiklehrkräften erfasst werden. Ersteres geschieht anhand öffentlich zugänglicher Dokumente der Hochschulen. Zur Erfassung des FW und FDW wurden Daten aus dem Projekt Kompetenzentwicklung in mathematischen und naturwissenschaftlichen Lehramtsstudiengängen (Akronym: KeiLa) genutzt, dessen Ziel es war, die Entwicklung des Professionswissens angehender Lehrkräfte verschiedener Fächer zu untersuchen. Zudem wird angenommen, dass sich Studienstrukturen über alle Studienjahre erstrecken und daher in Zusammenhang mit dem FW und FDW von Probanden unabhängig vom Studienjahr stehen.

\subsection{Stichprobenbeschreibung}

Im Fach Physik nahmen 20 Hochschulen mit $N=107$ Lehramtsstudierenden (43\% weiblich) aus den ersten sechs Semestern teil. 37 der 107 Probanden nahmen die 
Abb. 1 Verteilung der Datenpunkte über die Studienjahre

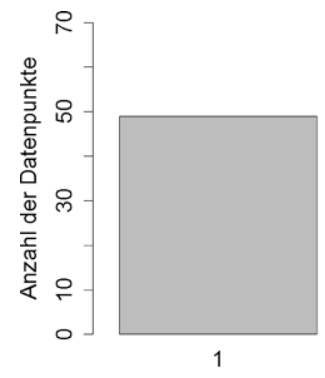

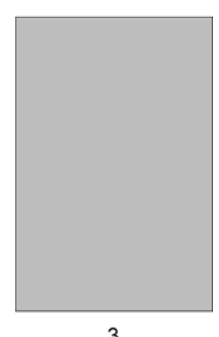

3

Studienjahr

Möglichkeit wahr, in den nachfolgenden Jahren wiederholt an der Messung des FW und FDW teilzunehmen. Für diesen Fall wurden die vorliegenden Längsschnittdaten der Studienteilnehmenden als Querschnittdaten interpretiert. Somit liegen von den $N=107$ Probanden insgesamt $n=159$ Datenpunkte vor. 88 Probanden (130 Datenpunkte) studieren mit dem Ziel des Gymnasiallehramts, die durchschnittliche Studiendauer beträgt 2,11 Jahre ( $S D=0,85$ Jahre). In Abb. 1 ist die Verteilung der Datenpunkt über die Studienjahre aufgeführt.

Die 20 teilnehmenden Hochschulen stammen aus elf verschiedenen Bundesländern, bei 3 der 20 Hochschulen handelt es sich um Pädagogische Hochschulen. Da einige Hochschulen das Studium verschiedener Schulformen anbieten (z. B. Gymnasiallehramt und Haupt- und Realschullehramt), liegen 25 Studiengänge aus 20 verschiedenen Hochschulen vor. Von den 25 Studiengängen sind 11 Studiengänge dem Staatsexamen-Modell zuzuordnen, die restlichen 14 Studiengänge folgen dem Bachelor-Master-Modell. Die Regelstudienzeit für das Physik-Lehramtsstudium beträgt für 2 Studiengänge 7 Semester, für 7 Studiengänge 8-9 Semester und für 16 Studiengänge 10 Semester. Die ausgewählten Hochschulen mit ihren Studiengängen decken somit die Breite unterschiedlicher regionaler Besonderheiten und Studienmodelle ab. Zudem verteilen sich die 107 Probanden (bzw. 159 Datenpunkte) relativ gleichmäßig über die 25 Studiengänge.

\subsection{Erfassung der Studienstrukturen}

Zur Erfassung der Studienstrukturen wurden die öffentlich zugänglichen Dokumente der 25 Studiengänge an 20 deutschen Hochschulen herangezogen. Waren innerhalb einer Hochschule Probanden verschiedener Schulformen vertreten (z. B. Gymnasiallehramt und Haupt- und Realschullehramt), so wurden beide Studiengänge getrennt erfasst. Außerdem führten 6 der 25 Studiengänge das sogenannte „Major-MinorModell“", in dem das Studium zweier Fächer nicht im gleichen Umfang geschieht. Für die Analyse der Studienstrukturen wurden für Studiengänge mit dem MajorMinor-Modell jeweils die Modulpläne für Physik als Hauptfach betrachtet.

Die Sammlung der Dokumente umfasste neben den Modulhandbüchern weiterhin Studienverlaufspläne und Studien- sowie Prüfungsordnungen. Die Dokumente entsprachen dabei dem Stand der Studienstruktur zu Beginn der Erhebung im Wintersemester 2014/2015. Mit Hilfe der gesammelten Dokumente wurde für jeden 
Studiengang eine tabellarische Übersicht aller Lehrveranstaltungen im Physik-Lehramtsstudium erstellt und entsprechend ihrer Thematik in Fach und Fachdidaktik unterschieden. Für Veranstaltungen, die Fach und Fachdidaktik gleichermaßen betrafen, wurde mit Hilfe der Angaben in den Modulhandbüchern entschieden, zu welchen Anteilen die Semesterwochenstunden (SWS) auf Fach und Fachdidaktik aufzuteilen sind. Waren in den Modulhandbüchern keine eindeutigen Informationen über die Aufteilung der SWS vorhanden, wurde anhand der Beschreibung der Modul-Lernziele überprüft, ob der Fokus der Veranstaltung eher auf fachwissenschaftliche oder fachdidaktische Inhalte lag. Hatten beispielsweise Experimentierpraktika Lernziele wie etwa Sachkompetenz in der Benutzung physikalischer Messgeräte, so wurden sie dem Fach zugeordnet. Fokussierten die Experimentierpraktika etwa das Kennenlernen von physikalischen Schulexperimenten, so wurden die SWS der Fachdidaktik zugeordnet. Darüber hinaus wurden Schulpraktika nicht berücksichtigt, da es sich hierbei um Lerngelegenheiten handelt, die unter anderem stark von der Praktikumsschule abhängig sind (z.B. die Betreuung durch Mentorinnen und Mentoren und Kollegium, Anzahl an Hospitationen, Anzahl an selbst erteilten Unterrichtsstunden) und weniger von den eigentlichen Studiengängen.

Zur Charakterisierung der Studieninhalte wurde mit Hilfe der tabellarischen Übersichten für jeden Studiengang erfasst, wie viele SWS ${ }^{1}$ jeweils auf Veranstaltungen der Experimentalphysik, der theoretischen Physik und der Fachdidaktik entfallen. Bei der Experimentalphysik und Fachdidaktik wurde zudem mit Hilfe des Modulhandbuches ermittelt, wie viele SWS für den jeweiligen Inhalt (gemäß Vorgaben der KMK 2019) vorgesehen waren. Für die Physikdidaktik ließen sich eine Vielzahl der fachdidaktischen Veranstaltungen (typischerweise die sog. Einführungsveranstaltungen) thematisch nicht eindeutig zuordnen, weshalb zusätzlich der Inhalt Allgemeine Fachdidaktik hinzugefügt wurde. Da die KMK (2019) für Studierende des Hauptund Realschullehramts im Bereich der theoretischen Physik lediglich eine „Übersicht über Strukturen und Konzepte der Physik“ (KMK 2019, S. 51) fordert, wurde bei der theoretischen Physik nicht nach einzelnen Inhalten unterschieden. Um nun Aussagen über das „typische“ Physik-Lehramtsstudium zu tätigen, wurden die inhaltsspezifischen SWS über alle Studiengänge gemittelt. Außerdem wurde ermittelt, wann der Inhalt durchschnittlich das erste Mal explizit thematisiert wird, mit wie vielen SWS dieser Inhalt maximal und minimal thematisiert wird und wie viele Studiengänge diesen Inhalt nicht explizit thematisieren.

Zur Beantwortung der zweiten Forschungsfrage wurde anhand der tabellarischen Übersichten berechnet, wie viele SWS Studierende bis zum Ende des sechsten Semesters in jedem der 25 Studiengänge in Fach und Fachdidaktik absolvieren sollten. Diese aufsummierten SWS in Fach und Fachdidaktik wurden dann genutzt, um entsprechende Cluster zu bilden. Ziel der Bildung von Clustern war es, die studiengangspezifischen SWS von Fach und Fachdidaktik so zu gruppieren (clustern), dass die Varianz innerhalb der Cluster minimal wird. Für die Bildung der Cluster

\footnotetext{
${ }^{1}$ In einigen Studien werden Leistungspunkte (LP) als Maß für die Quantität von Lehrveranstaltungen genutzt. Dabei ist zu beachten, dass LP u. a. auch individuelle Lernzeiten berücksichtigen. Da diese Studie jedoch die Strukturen des Studiums untersucht, werden die SWS als Maß für den Umfang von Lehrveranstaltungen genutzt.
} 
wurde die sogenannte k-Means-Methode (MacQueen 1967) gewählt. Die k-MeansMethode hat den Vorteil, dass jeder Cluster von einem ggf. fiktiven Zentrum (Centroid) repräsentiert wird (Kassambara 2017). Folglich liefert die k-Means-Methode neben den Clustern auch cluster-typische SWS für Fach und Fachdidaktik. Für die k-Means-Methode stehen verschiedene iterative Verfahren zu Verfügung (für einen Überblick siehe Sharafi 2013), welche die quadrierte (euklidische) Distanz zum Centroid minimieren. Für die vorliegende Studie wurde der Algorithmus von Hartigan und Wong (1979) genutzt, welcher im verwendeten R-Paket factoextra (Kassambara und Mundt 2017) implementiert ist. Zu beachten ist, dass es die k-MeansMethode verlangt, die gewünschte Clusteranzahl im Vorwege festzulegen (Hartigan und Wong 1979). Um die Frage nach der optimalen Clusteranzahl zu beantworten, wurde der durchschnittliche Silhouettenkoeffizient genutzt (Rousseeuw 1987). Der Silhouettenkoeffizient vergleicht für jedes Element die Entfernung von Elementen im eigenen Cluster (within) mit der Entfernung von Elementen in nächstliegenden Clustern (between). Der Silhouettenkoeffizient eines Elementes kann Werte zwischen -1 und 1 annehmen. Negative Werte (between-Abstände sind kleiner als within-Abstände) können als falsche Clusterzuordnung eines Elementes interpretiert werden, positive Werte (within-Abstände sind kleiner als between-Abstände) als gute Passung zwischen Element und Cluster. Beträgt der Silhouettenkoeffizient den Wert 0, so liegt ein Element genau zwischen zwei Clustern (Rousseeuw 1987). Um nun mit dem Silhouettenkoeffizienten die optimale Clusteranzahl $k$ zu finden, wird für verschiedene Werte für $k$ (z.B. von 1-15) der durchschnittliche Silhouettenkoeffizient aller Elemente bestimmt. Die Clusteranzahl $k$, für die der durchschnittliche Silhouettenkoeffizient maximal wird, kann dann als optimale Clusteranzahl interpretiert werden (Kassambara 2017). Auf diese Weise dient der durchschnittliche Silhouettenkoeffizient zum einen als Möglichkeit, die optimale Clusteranzahl zu bestimmen (Rousseeuw 1987) und zum anderen als eine von der Clusteranzahl unabhängige Maßzahl für die Qualität des Clusterings (Kassambara 2017).

\subsection{Erfassung des FW und FDW}

Die Testinstrumente für das FW und für das FDW der angehenden Physiklehrkräfte wurden im Projekt Messung professioneller Kompetenzen in mathematischen und naturwissenschaftlichen Lehramtsstudiengängen (Akronym: KiL) entwickelt und validiert (Sorge et al. 2019). Das Testinstrument für das FW der angehenden Physiklehrkräfte beinhaltet 40 Aufgaben (sogenannte Ankeritems) sowie zusätzlich 20/20/18 Aufgaben für Studierende des ersten/zweiten und dritten Studienjahres. Der Test adressiert die folgenden Inhaltsbereiche der Physik: Mechanik, Elektrodynamik, Optik, Thermodynamik, Festkörperphysik, Atom- und Kernphysik, spezielle Relativitätstheorie sowie Quantenphysik. Die Anzahl der Aufgabe je Inhaltsbereich schwankt jedoch abhängig vom studienjahrspezifischen Testheft. Von den 40 gemeinsamen Ankeritems liegen 33 Aufgaben als Multiple-Choice-Format vor. Das Testinstrument für das FDW der angehenden Physiklehrkräfte beinhaltet 39 Aufgaben und war für alle Probanden unabhängig ihres Studienjahres identisch. Der Test umfasst die Facetten Vorstellungen der Schülerinnen und Schüler, Instruktionsstrategien, Curriculum und Assessment (Magnusson et al. 1999) in den oben genannten 
physikalischen Inhaltsbereichen. Die gewählten Aufgabenformate (19 offene Aufgaben, 15 Aufgaben im Multiple-Choice-Format, 2 Wahr-Falsch-Aufgaben sowie 3 Zuordnungsaufgaben) waren über die Facetten vergleichbar verteilt.

Für die statistische Analyse, inwieweit die Studienstrukturen das FW und FDW angehender Physiklehrkräfte beeinflussen, wurden die Rohdaten der N=107 Probanden mit Hilfe des Rasch-Modells in Personenfähigkeiten überführt. Hierfür wurden virtuelle Personen genutzt, ,d.h. die Messungen derselben Personen zu späteren Zeitpunkten werden behandelt, als ob es sich um zusätzliche Personen handeln würde, die dieselben Items bearbeitet haben“ (Hartig und Kühnbach 2006, S. 33). Zum Teil vorliegende Längsschnittdaten wurden also als Querschnittdaten interpretiert, somit lagen $n=159$ Datenpunkte zur Analyse vor. Die Ergebnisse des fachwissenschaftlichen und fachdidaktischen Tests wurden mit dem R-Paket TAM (Robitzsch et al. 2019) analysiert, wobei die Tests jeweils simultan raschskaliert wurden (Trendtel et al. 2016). Fünf bzw. zwei Aufgaben des fachwissenschaftlichen bzw. fachdidaktischen Tests zeigten einen schlechten Infit (d.h. kleiner als 0,8 oder größer als 1,2 ) und wurden folglich aus der Analyse ausgeschlossen. Nach erneuter Raschskalierung zeigten die übrigen Items gute Infits (d.h. zwischen 0,8 und 1,2), was für eine hohe Passung zum gewählten Rasch-Modell spricht (Bond und Fox 2007). Beide Tests wiesen sehr gute WLE-Reliabilitäten auf $\left(\alpha_{\mathrm{WLE}, \mathrm{FW}}=0,88 ; \alpha_{\mathrm{WLE}, \mathrm{FDW}}=0,87\right)$. Die aus der Rasch-Analyse generierten Personenfähigkeiten für das FW und FDW wurden dann genutzt, um den Einfluss der Studienstrukturen näher zu bestimmen. Hierfür wurden verschiedene lineare Regressionsmodelle berechnet, die das FW und FDW der angehenden Physiklehrkräfte mit Hilfe der Clusterzugehörigkeit erklären sollen.

\section{Ergebnisse}

\subsection{Inhaltliche Beschreibung der Studienstrukturen}

Entsprechend der ersten Forschungsfrage wird im Folgenden dargestellt, wie das Physik-Lehramtsstudium in Deutschland in den ersten sechs Studiensemestern strukturiert ist.

Die Analyse der Dokumente der 25 Studiengänge zeigt, dass bis zum Ende des sechsten Semesters im Durchschnitt 44,32 SWS ( $S D=13,83$ SWS) im Fach und 9,46 SWS ( $S D=4,55$ SWS) in Fachdidaktik vorgesehen sind. Im Durchschnitt ist also die Zahl der SWS, die auf fachliche Veranstaltungen entfallen, 4,7-mal höher als die der SWS, die auf fachdidaktische Veranstaltungen entfallen (vgl. Abb. 2). Diese Beobachtung könnte jedoch durch die Entscheidung, nur die ersten sechs Semester des Physik-Lehramtsstudiums zu betrachten, bedingt sein.

Um Aussagen über Häufigkeit und Sequenzierung der Inhalte zu treffen, wurden für jeden der 25 Studiengänge die SWS entlang der von der KMK (2019) definierten Themenbereiche Experimentalphysik, theoretische Physik und Fachdidaktik aufsummiert (Abb. 3).

In Abb. 3 fällt zunächst auf, dass im Mittel die meisten SWS auf die theoretische Physik entfallen; wobei dieser Wert auch die höchste Varianz aufweist. Beides hängt 


\begin{abstract}
Abb. 2 Boxplot der aufsummierten Semesterwochenstunden (SWS) in den 25 Studiengänge bis zum Ende des sechsten Semesters. Links (grün) sind die SWS im Fach, rechts (orange) die SWS in der Fachdidaktik dargestellt. Die Box spannt sich jeweils vom 25\%-Quantil bis zum $75 \%$-Quantil auf, der Balken in der Mitte der Box kennzeichnet den jeweiligen Median
\end{abstract}

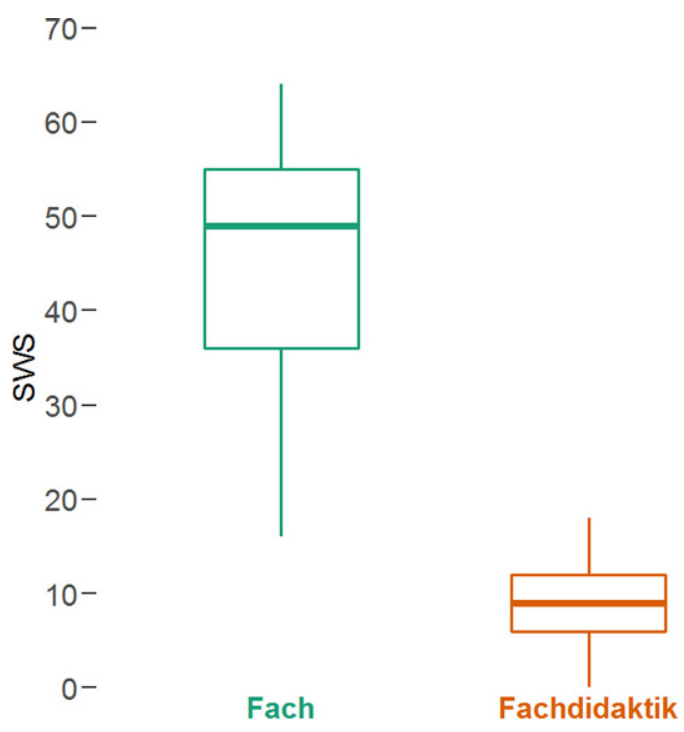

jedoch mit der Entscheidung zusammen, die theoretische Physik nicht in Inhaltsbereiche zu differenzieren. Der Inhaltsbereich Elektrodynamik und Optik hat nach der theoretischen Physik die meisten SWS, die größte Variation nach der theoretischen Physik weist der Inhaltsbereich Festkörper-, Kern- und Elementarteilchenphysik, Kosmologie auf. Die wenigsten SWS werden im Inhaltsbereich Thermodynamik absolviert. Die Inhaltsbereiche Mechanik sowie Elektrodynamik und Optik werden in allen 25 Studiengängen mit mindestens 2 SWS fokussiert. Aus dem durchschnittlichen Startzeitpunkt lässt sich zudem ableiten, dass die Inhaltsbereiche Mechanik und Thermodynamik vorzugsweise im selben Semester fokussiert werden (analog zum Inhaltsbereich Elektrodynamik und Optik). Unterstützt wird diese Folgerung durch die mittleren SWS der Elektrodynamik und Optik, die vergleichbar mit den summierten SWS von Mechanik und Thermodynamik sind.

Für die Inhalte der fachdidaktischen Ausbildung belegen die Ergebnisse in Abb. 3, dass sie deutlich weniger kanonisch verläuft als die fachliche Ausbildung. Es zeigt sich aber auch, dass bis zum Ende des sechsten Semesters typischerweise allgemeine Fachdidaktik (in der Regel in Form von sog. Einführungsveranstaltungen) gelehrt wird. Außerdem wird in einer Vielzahl von Studiengängen (16 von 25) das Hauptaugenmerk auf die Planung und Analyse von Physikunterricht gelegt. Immerhin 10 Studiengänge explizieren Aufgaben, Experimente und Medien in ihrer fachdidaktischen Ausbildung. Motivation und Interesse, fachdidaktische Positionen und Konzeptionen sowie die fachdidaktische Forschung werden in einer Vielzahl von Studiengängen zunächst nicht thematisiert. Verlässliche Aussagen zu einer ,typischen" Abfolge der fachdidaktischen Ausbildung können aufgrund der geringen SWS der Inhalte nicht getätigt werden. Jedoch stärkt Abb. 3 den Eindruck, dass die fachdidaktische Ausbildung typischerweise im dritten und vierten Semester stattfindet. 


\begin{tabular}{lllllll}
\hline Inhalt & $M$ & $S D$ & Min & Max & Start & NA \\
\hline Mechanik & 4,10 & 1,85 & 2 & 10 & 1 & 0 \\
Thermodynamik & 3,29 & 1,33 & 0 & 7 & 1 & 1 \\
Elektrodynamik und Optik & 6,65 & 2,40 & 2 & 12 & 2 & 0 \\
Atom- und Quantenphysik & 5,21 & 3,16 & 2 & 16 & 4 & 2 \\
Festkörper-, Kern- und Elementarteilchenphysik, & 4,79 & 3,66 & 0 & 12 & 5 & 7 \\
Kosmologie & 8,33 & 6,70 & 0 & 22 & 3 & 9 \\
Theoretische Physik & 0,83 & 1,55 & 0 & 6 & 4 & 19 \\
Fachdidaktische Positionen und Konzeptionen & 0,08 & 0,41 & 0 & 2 & 3 & 24 \\
Motivation und Interesse & 0,42 & 0,78 & 0 & 2 & 3,5 & 19 \\
Lernprozesse, Diagnose von Lernschwierigkeiten & 2,00 & 1,79 & 0 & 6 & 4 & 9 \\
Planung und Analyse von Physikunterricht & 1,17 & 1,37 & 0 & 4 & 4 & 14 \\
Aufgaben, Experimente und Medien & 0,25 & 0,68 & 0 & 2 & 5,5 & 23 \\
Fachdidaktische Forschung & 2,08 & 2,00 & 0 & 8 & 3 & 6 \\
Allgemeine Fachdidaktik & & & & & & \\
\hline
\end{tabular}

Abb. 3 Mittlere SWS (bis zum Ende des sechsten Semesters) über alle 25 Studiengänge entlang der Themenbereiche (dargestellt sind die Themenbereiche Experimentalphysik (Inhalte 1-5, grün), theoretische Physik und Physikdidaktik (Inhalte 7-13, orange). $M$ gibt den durchschnittlichen Umfang in SWS an, SD die Standardabweichung. Min bzw. Max stellt die kleinste bzw. größte SWS-Anzahl über alle 25 Studiengänge dar. Start repräsentiert den Median der Semester, in dem der Inhalt das erste Mal in einer Veranstaltung fokussiert wird. NA gibt an, wie viele Studiengänge den Inhalt bis zum Ende des sechsten Semesters nicht adressieren

Abb. 3 gibt insgesamt einen Überblick, welche Inhalte in welcher Intensität in den ersten sechs Semestern des Physik-Lehramtsstudiums thematisiert werden. Jedoch zeigt sich in den teilweise hohen Standardabweichungen, dass es bedeutende Variationen zwischen den einzelnen Studiengängen gibt.

\subsection{Clusterung der Studiengänge}

Um die verschiedenen Physik-Lehramtsstudiengänge systematisch zu gruppieren, wurden die aufsummierten SWS in Fach und Fachdidaktik der 25 Studiengänge genutzt, um entsprechende Cluster zu bilden. Da sich die aufsummierten SWS in Fach und Fachdidaktik jedoch stark in ihrer Größe unterscheiden, wurden für die Clusteranalyse der Studiengänge die aufsummierten SWS von Fach und Fachdidaktik z-standardisiert. Die Daten der z-standardisierten SWS aller Studiengänge zeigen für $k=4$ Cluster den maximalen durchschnittlichen Silhouettenkoeffizienten von 0,52. Dies kann zum einen als eine angemessene Qualität der Clusterung interpretiert werden (Kaufman und Rousseeuw 1990) und es kann zum anderen angenommen werden, dass die Daten durch vier Cluster am besten beschrieben werden (Kaufman und Rousseeuw 1990). Das Ergebnis der Clusterung ist in Abb. 4 dargestellt. Durch diese Cluster können 77,4\% der Varianz innerhalb der SWS von Fach bzw. Fachdidaktik auf den Unterschied zwischen den Clustern zurückgeführt werden. Anhand der Centroide in Abb. 4 können die cluster-typischen SWS für Fach und Fachdidaktik bestimmt werden, diese sind in Tab. 1 aufgeführt. Hieran kann abgelesen werden, dass die Cluster 1 und 2 sowie 3 und 4 vergleichbare SWS im Fach aufweisen. 


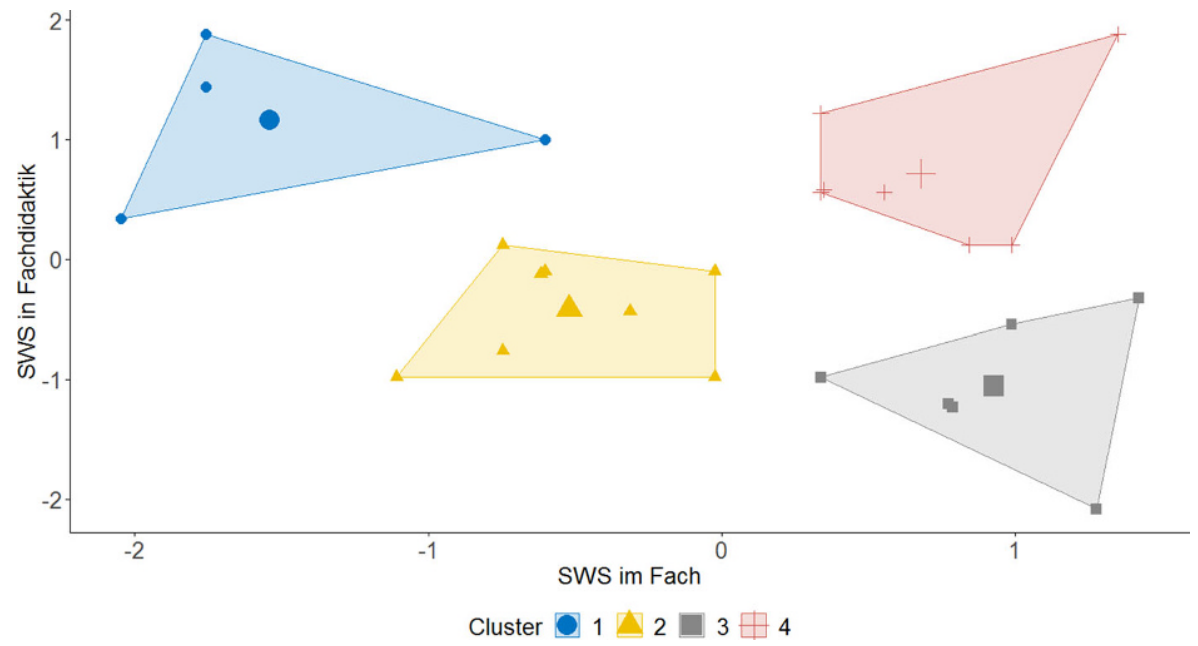

Abb. 4 Ergebnis der k-mean Clusterung mit $k=4$ Cluster gemäß ihrer Semesterwochenstunden (SWS) in Fach und Fachdidaktik bis zum Ende des sechsten Semesters. Die großen Symbole repräsentieren den jeweiligen Centroid des Clusters. Die x- und y-Achse sind z-standardisiert

Tab. 1 Centroide der vier Cluster

\begin{tabular}{lll}
\hline Cluster & Mittlere SWS Fach & Mittlere SWS Fachdidaktik \\
\hline 1 & $23(8,87)$ & $14,75(2,99)$ \\
2 & $37,13(5,22)$ & $7,56(1,99)$ \\
3 & $57,17(5,42)$ & $4,67(2,80)$ \\
4 & $53,71(5,47)$ & $12,71(2,87)$ \\
\hline
\end{tabular}

Dargestellt sind die jeweiligen fiktiven Zentren (d.h. die SWS in Fach und Fachdidaktik gemittelt über alle im Cluster befindlichen Studiengänge) der vier Cluster

Die Klammer enthält die jeweilige Standardabweichung

Hingegen zeigen die Cluster 1 und 4 sowie 2 und 3 vergleichbare SWS in der Fachdidaktik. Diese Unterscheidung wird dadurch bestätigt, dass sich die Clusterpaare vergleichbarer SWS tatsächlich signifikant in der Gesamtanzahl an fachlichen bzw. fachdidaktischen SWS voneinander unterscheiden (Fach: $F(1,23)=56,81 ; p<0,001$; $\eta^{2}=0,71 ;$ Fachdidaktik: $\left.F(1,23)=39,49 ; p<0,001 ; \eta^{2}=0,63\right)$.

Teilt man das Koordinatensystem in Abb. 4 in die vier Quadranten auf, lässt sich jeder der vier Cluster genau einem Quadranten zuordnen. Da beide Achsen z-standardisiert sind, der Ursprung beider Achsen also dem Mittelwert der SWS entspricht, kann man Cluster 1 und 2 einen geringen Fachanteil, Cluster 3 und 4 einen umfangreichen Fachanteil, Cluster 2 und 3 einen geringen Fachdidaktikanteil und Cluster 1 und 4 einen umfangreichen Fachdidaktikanteil zuordnen.

\subsection{Einfluss der Cluster auf das FW und FDW}

Zur Beantwortung der dritten Forschungsfrage wurde der Einfluss der Studienstrukturen auf das FW und FDW der angehenden Physiklehrkräfte untersucht. Dazu 
Tab. 2 Korrelationsmatrix und deskriptive Angaben der verwendeten Variablen

\begin{tabular}{lllllllll}
\hline & $\boldsymbol{M}$ & $\mathbf{S D}$ & $\mathbf{( 2 )}$ & $\mathbf{( 3 )}$ & $\mathbf{( 4 )}$ & $\mathbf{( 5 )}$ & $\mathbf{( 6 )}$ & $\mathbf{( 7 )}$ \\
(1) Abitur & 2,19 & 0,59 & 0,00 & $-0,01$ & $-0,33^{* * *}$ & $-0,36^{* * *}$ & $0,18^{*}$ & 0,12 \\
(2) Studienjahr & 2,11 & 0,85 & - & $-0,01$ & $0,44^{* * *}$ & $0,38^{* * *}$ & $-0,01$ & $-0,02$ \\
(3) Geschlecht & - & - & - & - & $0,38^{* * *}$ & 0,12 & $-0,09$ & $-0,22^{* *}$ \\
(4) FW & - & - & - & - & - & $0,58^{* * *}$ & $-0,26^{* * *}$ & $-0,20^{* *}$ \\
(5) FDW & - & - & - & - & - & - & $-0,23^{* *}$ & $-0,05$ \\
(6) Geringer & - & - & - & - & - & - & - & $0,20^{* *}$ \\
Fachanteil & & & & & & & - & - \\
(7) Geringer & - & - & - & - & - & & & - \\
Fachdidaktik- & & & & & & & & \\
anteil & & & & & & & & \\
\hline
\end{tabular}

$M$ gibt den Mittelwert der jeweiligen Skala an, SD die Standardabweichung

Geringer Fach- bzw. Fachdidaktikanteil wurde bei Zugehörigkeit zum Cluster 1 oder 2 bzw. 2 oder 3 mit 1 codiert, ansonsten mit 0 ; Geschlecht: $0=$ weiblich, 1 = männlich

FW und FDW sind aus dem Rasch-Modell resultierende Personenfähigkeiten, liegen also auf einer willkürlichen Skala, somit sind Mittelwerte und Standardabweichungen nicht interpretierbar

Für dichotome Variablen wurde darauf verzichtet, Mittelwerte und Standardabweichungen anzugeben ${ }^{*} p<0,05 ;{ }^{* *} p<0,01 ;{ }^{* * *} p<0,001$

wurde zunächst ein Regressionsmodell mit der Abiturnote, dem Geschlecht sowie dem aktuellen Studienjahr als Prädiktoren für das FW und FDW als Baseline-Modell geschätzt. Diese individuellen Faktoren haben sich in der bisherigen Literatur als bedeutsam für das FW und FDW herausgestellt (z. B. Reinhold und Riese 2012; Sorge et al. 2019; Woitkowski 2020). Anschließend wurden die Cluster als Maß der Studienstruktur als Prädiktor für das FW und FDW hinzugefügt. Dadurch wird berücksichtigt, dass das fachspezifische Lehramtsstudium (also die fachliche und fachdidaktische Ausbildung) zwei-dimensional vorliegt und beide Ausbildungen abhängig voneinander sind. Deskriptive Angaben sowie die Korrelationen zwischen den untersuchten Variablen sind der Tab. 2 zu entnehmen. Es ist zu erkennen, dass die Abiturnote und das Studienjahr signifikant mit dem FW und FDW korrelieren. Zudem korrelieren Geschlecht (männlich) und FW signifikant. Anhand von Tab. 2

Tab. 3 Regressionsmodelle zur Erklärung des Fachwissens angehender Physiklehrkräfte

\begin{tabular}{lllll}
\hline & Modell 1 & \multicolumn{3}{c}{ Modell 2} \\
& $\beta$ & $p$ & $\beta$ & $p$ \\
\hline Achsenabschnitt & 0,00 & 0,328 & 0,00 & 0,267 \\
Abitur & $-0,33$ & $0,000^{* * *}$ & $-0,29$ & $0,000^{* * *}$ \\
Studienjahr & 0,45 & $0,000^{* * *}$ & 0,44 & $0,000^{* * *}$ \\
Geschlecht & 0,37 & $0,000^{* * *}$ & 0,35 & $0,000^{* * *}$ \\
Geringer Fachanteil & - & - & $-0,16$ & $0,008^{* *}$ \\
Geringer Fachdidaktikanteil & - & - & $-0,05$ & 0,455 \\
$\mathrm{R}^{2}$ & 0,45 & - & 0,48 & - \\
\hline
\end{tabular}

Abgebildet sind die standardisierten Regressionskoeffizienten

Geringer Fach- bzw. Fachdidaktikanteil wurde bei Zugehörigkeit zum Cluster 1 oder 2 bzw. 2 oder 3 mit 1 codiert, ansonsten mit 0 ; Geschlecht: $0=$ weiblich, $1=$ männlich ${ }^{* *} p<0,01 ;{ }^{* * *} p<0,001$ 
Tab. 4 Regressionsmodelle zur Erklärung des fachdidaktischen Wissens angehender Physiklehrkräfte

\begin{tabular}{|c|c|c|c|c|c|c|}
\hline & \multicolumn{2}{|c|}{ Modell 1} & \multicolumn{2}{|c|}{ Modell 2} & \multicolumn{2}{|c|}{ Modell 3} \\
\hline & $\beta$ & $p$ & $\beta$ & $p$ & $\beta$ & $p$ \\
\hline Achsenabschnitt & 0,00 & 0,073 & 0,00 & 0,115 & 0,00 & $0,021^{*}$ \\
\hline Abitur & $-0,20$ & $0,003^{* *}$ & $-0,20$ & $0,004^{* *}$ & $-0,21$ & $0,003^{* *}$ \\
\hline Studienjahr & 0,17 & $0,019^{*}$ & 0,18 & $0,014^{*}$ & 0,14 & 0,056 \\
\hline Geschlecht & $-0,05$ & 0,470 & $-0,03$ & 0,625 & $-0,05$ & 0,464 \\
\hline FW & 0,45 & $0,000^{* * *}$ & 0,43 & $0,000^{* * *}$ & 0,30 & $0,004^{* *}$ \\
\hline Geringer Fachanteil & - & - & $-0,10$ & 0,137 & $-0,09$ & 0,173 \\
\hline Geringer Fachdidaktikanteil & - & - & 0,07 & 0,271 & $-0,02$ & 0,781 \\
\hline Geringer Fachanteil: FW & - & - & - & - & 0,07 & 0,369 \\
\hline $\begin{array}{l}\text { Geringer Fachdidaktikanteil: } \\
\text { FW }\end{array}$ & - & - & - & - & 0,21 & $0,020^{*}$ \\
\hline $\mathrm{R}^{2}$ & 0,40 & - & 0,41 & - & 0,44 & - \\
\hline
\end{tabular}

Abgebildet sind die standardisierten Regressionskoeffizienten Geringer Fach- bzw. Fachdidaktikanteil wurde bei Zugehörigkeit zum Cluster 1 oder 2 bzw. 2 oder 3 mit 1 codiert, ansonsten mit 0 ; Geschlecht: $0=$ weiblich, $1=$ männlich ${ }^{*} p<0,05 ;{ }^{* *} p<0,01 ;{ }^{* * *} p<0,001$

ist außerdem zu erkennen, dass das FW und FDW der Probanden signifikant zusammenhängen und abhängig von der Clusterzugehörigkeit sein könnten.

Für das FW (Tab. 3) klärt das Regressionsmodell mit Cluster (Modell 2) im Vergleich zum Referenzmodell (Modell 1) signifikant mehr Varianz in den Daten auf $(F(2,151)=4,33 ; p=0,015)$, somit lässt sich das FW der angehenden Physiklehrkräfte signifikant besser durch den Einbezug der Studienstruktur erklären. Anhand der standardisierten Regressionsgewichte aus Tab. 3 ist erkennbar, dass sich Cluster mit umfangreichem Fachanteil signifikant besser auf das FW der Probanden auswirken als Cluster mit geringem Fachanteil $(\beta=-0,16 ; p=0,008)$.

Für das FDW (Tab. 4) ist zu erkennen, dass das Modell 2 nicht signifikant mehr Varianz in den Daten aufklärt als das Referenzmodell $(F(2,150)=1,52 ; p=0,22)$. Somit ist zunächst anzunehmen, dass das FDW der Probanden nicht signifikant besser durch die alleinige Hinzunahme der Studienstruktur erklärt wird. Jedoch zeigt sich, dass das FDW der Probanden stark durch das FW vorhergesagt wird (im Modell 1: $\beta=0,45 ; p<0,001$ ). Modell 3 (Tab. 4) bezieht deshalb mögliche Interaktionseffekte zwischen FW und Cluster ein. Zu erkennen ist, dass Modell 3 signifikant mehr Varianz in den Daten aufklärt als Modell $1(F(4,148)=2,65 ; p=0,035)$, somit lässt sich auch das FDW der angehenden Physiklehrkräfte signifikant besser unter Einbezug der Interaktion von FW und Cluster erklären. An den Regressionskoeffizienten in Tab. 4 ist ersichtlich, dass Cluster mit geringem Fach- wie auch Fachdidaktikanteil allein keinen direkten Einfluss auf das FDW besitzen (Fach: $\beta=-0,09 ; p=0,173$; Fachdidaktik: $\beta=-0,02 ; p=0,781)$. Jedoch zeigt sich neben dem positiven Einfluss des FW auf das FDW, dass ein signifikanter Interaktionseffekt von Clustern mit geringem Fachdidaktikanteil und FW auf das FDW besteht $(\beta=0,21 ; p=0,020)$. Anders formuliert zeigt dieses Ergebnis, dass der ohnehin positive Effekt des FW auf das FDW in den Clustern mit geringem Fachdidaktikanteil noch verstärkt wird. 


\section{Diskussion}

Die Entwicklung des FW und FDW angehender Lehrkräfte erfolgt maßgeblich in der universitären Lehramtsausbildung (z.B. Anders und Kleickmann 2011). Trotz der Bologna-Reform und bundesweiten Standards herrscht an deutschen Hochschulen aktuell jedoch ein hohes Maß an Heterogenität in den Lehramtsstudiengängen (z. B. Keller 2010; Keuffer 2010). Der Zusammenhang zwischen diesen unterschiedlichen Studienstrukturen und dem Professionswissen angehender Physiklehrkräfte ist bislang weitgehend unklar. Ziel des vorliegenden Artikels war es deshalb, die intendierten Curricula der universitären Lehramtsausbildung angehender Physiklehrkräfte systematisch zu analysieren und mit dem erreichten Curriculum im FW und FDW zu verknüpfen. Die systematische Analyse der öffentlich zugänglichen Dokumente von 25 Studiengängen an 20 deutschen Hochschulen zeigte vier typische Studienstrukturen. Diese vier Studienstrukturen können dabei jeweils einem geringen oder umfangreichen Fach- bzw. Fachdidaktikanteil zugeordnet werden. Darüber hinaus konnte gezeigt werden, dass Probanden in Clustern mit umfangreichem Fachanteil ein signifikant höheres FW aufweisen, wohingegen das FDW von Studierenden aus Studiengängen mit geringem Fachdidaktikanteil zu einem großen Teil auf deren FW und den entsprechenden fachlichen Lerngelegenheiten beruht. Somit konnte gezeigt werden, wie strukturelle Unterschiede in den Studiengängen im Zusammenhang mit dem FW und FDW der angehenden Physiklehrkräfte stehen. Die Ergebnisse der curricularen Analyse verdeutlichen auch, dass der Umfang an fachwissenschaftlichen Veranstaltungen den der fachdidaktischen Veranstaltungen bis zum Ende des sechsten Semesters im Schnitt um das Fünffache übertrifft. Es sei jedoch erwähnt, dass dieses Ergebnis mit der methodischen Entscheidung einhergeht, nur die ersten sechs Semester des Physik-Lehramtsstudiums zu betrachten. Gerade in sequenziellen Bachelor-Master-Studienmodellen sollten vermehrt eher fachwissenschaftliche Veranstaltungen im Bachelor-Studium stattfinden. Dennoch findet eine, wenn auch vergleichsweise geringe, fachdidaktische Ausbildung statt. Somit erfahren die angehenden Physiklehrkräfte bereits innerhalb der ersten sechs Semester eine lehramtsspezifische Ausbildung, was bedeutet, dass das in Deutschland vorliegende Lehramtsstudium nicht sequenziell sondern eher integrativ (Terhart 2008) verläuft.

Bezüglich der fachwissenschaftlichen Ausbildung lässt sich erkennen, dass die Fachinhalte Mechanik und Elektrodynamik und Optik in jedem der 25 Studiengänge explizit gelehrt werden. Zudem werden die Bereiche Mechanik und Thermodynamik typischerweise in derselben Veranstaltung bzw. im selben Modul behandelt. Der Umfang der theoretischen Physik weist die höchste Zahl an SWS aber auch die stärkste Variation auf. Beides hängt mit der Entscheidung zusammen, die theoretische Physik nicht in Inhaltsbereiche zu differenzieren, um die Vergleichbarkeit gymnasialer und nicht-gymnasialer Studiengänge zu erhöhen. Mithilfe der inhaltlichen Differenzierung der Studiengänge kann ein typischer Studienverlauf in der fachlichen Ausbildung festgestellt werden: Mechanik und Thermodynamik; Elektrodynamik und Optik; Atom- und Quantenphysik; Festkörper-, Kern- und Elementarteilchenphysik, Kosmologie. Die theoretische Physik beginnt typischerweise mit dem dritten Semester. Vergleicht man den hier identifizierten Studienverlauf mit den Empfehlungen der DPG (2014), so empfiehlt die DPG (2014) analog zu den Ergebnissen dieser 
Studie, die Inhalte Mechanik und Thermodynamik gemeinsam im ersten Semester anzubieten, Elektrodynamik im zweiten Semester und Quanten- und Atomphysik im vierten Semester zu verorten. Jedoch trennt die DPG (2014) die Inhaltsbereich Optik und Elektrodynamik voneinander und schlägt zwei neue „Inhaltspaare“ vor: Elektrodynamik und Relativität im zweiten Semester sowie Optik und Quantenphysik im dritten Semester. Zudem wird der Inhaltsbereich Festkörper-, Kern- und Elementarteilchenphysik, Kosmologie am Ende des Lehramtsstudiums (Semester 8, 9 und 10) verortet. Es ist also durchaus möglich, dass bei den Studiengängen, die diese Inhalte in den ersten sechs Semestern nicht behandelt haben, diese später noch adressiert werden.

Die fachdidaktische Ausbildung bis zum Ende des sechsten Semesters verläuft wesentlich weniger kanonisch. Größtenteils werden die von der KMK (2019) vorgegeben Inhaltsbereiche in den Dokumenten nicht explizit adressiert. Auffällig ist, dass der Planung und Analyse von Physikunterricht eine herausragende Stellung in der fachdidaktischen Ausbildung zukommt, da die meisten der untersuchten Studiengänge (ausschließlich) diesen Inhaltsbereich explizit in den Curricula erwähnen. Die übrigen fachdidaktischen Veranstaltungen sind oft nicht einem speziellen Inhaltsbereich zuzuordnen, sodass sie eher als eine überblicksartige Einführungsveranstaltung zu interpretieren sind. Dies kann durchaus als problematisch gewertet werden, da es nach Kind (2009) unverzichtbar ist, dass (angehende) Lehrkräfte die Inhalte der Fachdidaktik (neben denen der Fachwissenschaft) explizit erfahren (,,be more aware of the process they are undertaking“, Kind 2009, S. 170). Folglich sollte das Lehramtsstudium als Grundlage der fachdidaktischen Entwicklung die von der KMK (2019) als relevant eingestuften Inhaltsbereiche explizit fokussieren und deren Bedeutung für das FDW thematisieren (,making PCK more explicit in the teacher education process“, Kind 2009, S. 169), auch, um die fachdidaktische Ausbildung zwischen Studiengängen vergleichbar zu machen. Da sich die vorliegenden Analysen jedoch auf die ersten sechs Semester konzentrieren, ist es durchaus denkbar und zu erwarten, dass die Hochschulen diese Aufgabe im zweiten Teil des Lehramtsstudiums erfüllen.

In den Regressionsmodellen zeigte sich, dass die Zugehörigkeit zu einem Cluster allein keinen signifikanten Effekt auf das FDW der Probanden hat. Grund dafür könnte sein, dass selbst in Clustern mit umfangreichem Fachdidaktikanteil die Anzahl an Lerngelegenheiten zu niedrig ist und somit kein Effekt nachweisbar war. In Clustern mit umfangreichem Fachdidaktikanteil beträgt die durchschnittliche Anzahl der SWS in der Fachdidaktik 13,45 SWS. Zum Vergleich werden selbst in Clustern mit geringem Fachanteil noch durchschnittlich 32,42 SWS im Fach angeboten. Allerdings konnte festgestellt werden, dass das FW einen signifikanten Einfluss auf die Höhe des FDW besitzt. Auch dieses Ergebnis bestätigt vorangegangene empirische Untersuchungen, die den positiven Effekt des FW auf das FDW hervorheben (z. B. Baumert et al. 2010; Großschedl et al. 2015). Darüber hinaus zeigte sich, dass die Interaktion zwischen Clustern mit geringem Fachdidaktikanteil und FW signifikant auf das FDW angehender Physiklehrkräfte Einfluss nimmt. Somit legen unsere Daten nahe, dass die Anzahl an fachdidaktischen Veranstaltungen den Einfluss des FW auf das FDW moderiert. Im Falle von wenig fachdidaktischen Veranstaltungen ist zu erkennen, dass sich die Höhe des FW der angehenden Physiklehrkräfte signifi- 
kant auf deren FDW auswirkt. Sind hingegen viele fachdidaktische Veranstaltungen vorgesehen, so wirkt sich verschieden stark ausgeprägtes FW nicht unterschiedlich auf das FDW aus. Daraus lässt sich ableiten, dass vor allem bei wenig fachdidaktischen Veranstaltungen bis zum Ende des sechsten Semesters die Höhe des FW entscheidend für das FDW der angehenden Physiklehrkräfte ist. Ein Grund für diese Interaktion könnte sein, dass bei einem hohen Fachdidaktikanteil mehr Lernzeit zur Verfügung steht, um grundlegende fachdidaktische Prinzipien unabhängig vom Fachwissen zu vermitteln. Die hier gefundenen Ergebnisse betonen also ein weiteres Mal die Bedeutsamkeit des FW (und der fachlichen Veranstaltungen) für ein angemessenes FDW - besonders in Studiengängen, in denen wenig fachdidaktische Veranstaltungen angeboten werden und das FDW der Lehramtsstudierenden weniger explizit adressiert wird.

Bezüglich des Zusammenhangs von Studienstruktur und Fachwissen angehender Physiklehrkräfte legen die Ergebnisse nahe, dass angehende Physiklehrkräfte in Clustern mit geringem Fachanteil ein signifikant schlechteres FW aufweisen als jene in Clustern mit umfangreichem Fachanteil. Diese Beobachtung stimmt im Wesentlichen mit anderen empirischen Untersuchungen überein, die einen Einfluss der Quantität von Lerngelegenheiten auf das FW fanden (z.B. Großschedl et al. 2015; Reinhold und Riese 2012; Schödl 2017; Sorge et al. 2019). An dieser Stelle sei jedoch hervorgehoben, dass hier, im Gegensatz zu vorherigen Studien, zwei verschiedene Datenquellen verknüpft wurden: auf der einen Seite die Modulpläne der Hochschulen, auf der anderen Seite die Testergebnisse der Probanden. Somit zeigt dieses Ergebnis, dass es möglich ist, die intendierten Curricula mit den erreichten Curricula zumindest in Teilen in Einklang zu bringen. Dies legt zudem die Vermutung nahe, die Umsetzung und Nutzung der Lernangebote (also die implementierten Curricula) als ebenso wirkungsvoll zu klassifizieren.

Mit Blick auf die Ergebnisse ist jedoch zu beachten, dass die erreichten Curricula ausschließlich durch Paper-Pencil-basierte Wissenstests operationalisiert wurden. Das fachdidaktische Können, wie es etwa im Refined Consensus Model beschrieben wird (Carlson und Daehler 2019), wurde nicht mit einbezogen. In den letzten Jahren konnte gezeigt werden, dass sogenannte Performanztests ein valides Messinstrument darstellen, um eine handlungsnahe Kompetenz von Lehrkräften abzubilden (z. B. Kulgemeyer und Riese 2018). Jedoch wird im Modell von Carlson und Daehler (2019) auch hervorgehoben, dass das Wissen als Voraussetzung für Können verstanden werden muss, FW und FDW somit die Grundlage für handlungsnahe Kompetenzen darstellen (auch Blömeke et al. 2015). Darüber hinaus ist es aktuell unklar, durch welche Lerngelegenheiten angehende Lehrkräfte tatsächlich handlungsnahe Kompetenzen erwerben. Erste Ergebnisse lassen darauf schließen, dass Praktika bzw. Praxissemester bedeutende Gelegenheiten hierfür darstellen (z.B. Schröder et al. 2020), die allerdings in dieser Studie nicht erfasst wurden. Nichtsdestotrotz erscheinen Performanztests für die Operationalisierung von fachdidaktischem Können vielversprechend zu sein, weshalb zukünftige Studien auch die Auswirkungen der universitären Lerngelegenheiten auf die Performanz angehender Lehrkräfte stärker in den Blick nehmen sollten.

Bei der Untersuchung und Beschreibung der Studiengänge hinsichtlich ihrer fachlichen und fachdidaktischen Anteile ist zu beachten, dass lediglich ein spezifischer 
Abschnitt des tatsächlichen Physik-Lehramtsstudiums untersucht wurde. Diese Einschränkung war nötig, da die Studiendauer für die verschiedenen Lehramts- und Hochschultypen stark variiert (Radhoff und Ruberg 2016). Beispielsweise umfasst die Studiendauer bei 2 der 25 Studiengänge sieben Semester. Um also alle Studiengänge und dabei eine möglichst lange Studiendauer abzudecken, wurden nur die ersten sechs Semester berücksichtigt. Es ist demnach denkbar, dass die fachdidaktischen Inhalte an den meisten Hochschulen vermehrt und vor allem explizit nach dem sechsten Semester gelehrt werden (insbesondere im Bachelor-Master-Modell). Trotzdem kann nicht erwartet werden, dass sich das Verhältnis von fachlichen und fachdidaktischen Anteilen in der zweiten Hälfte des Studiums umkehrt, sodass auch am Ende der universitären Ausbildung die fachlichen Anteile die der fachdidaktischen übersteigen werden. Darauf aufbauend sollten Forscherinnen und Forscher zukünftig die zweite Hälfte des Physik-Lehramtsstudiums untersuchen, um insbesondere mehr Erkenntnisse über die fachdidaktische Ausbildung von angehenden Physiklehrkräften zu erlangen. Dennoch bieten die hier vorgestellten Ergebnisse zumindest die Möglichkeit, die Rolle des ersten Teils des Lehramtsstudiums zu klären und sind somit als erster Beitrag zu verstehen, die Heterogenität in den Studienstrukturen sowie deren Einfluss auf das Professionswissen der Studierenden zu beschreiben.

Zudem liefern die Ergebnisse keine Aussagen über die individuelle Entwicklung der Lehramtsstudierenden. Wie sich die verschiedenen Studienstrukturen also auf die individuellen Wissenszuwächse auswirken, kann hier nicht beantwortet werden. Für zukünftige Forschungsvorhaben sollten außerdem weitere Faktoren des Lehramtsstudiums mit einbezogen werden. Zu beachten ist weiterhin, dass die Regressionsmodelle unter Hinzunahme der Studienstruktur nur eine geringe (aber signifikante) zusätzliche Varianzaufklärung bringen, was vermutlich in der Distanz zwischen intendierten und erreichten Curricula begründet liegt. Zukünftige Studien sollten verstärkt das implementierte Curriculum in den Blick nehmen, um den Übergang von intendierten $\mathrm{zu}$ erreichten Curricula genauer zu beleuchten. So könnten beispielsweise Befragungen der Dozierenden oder Beobachtungsstudien in formalen Lerngelegenheiten den Zusammenhang zwischen Studienstrukturen und Professionswissen weiter aufklären. Dennoch stellt die vorliegende Studie einen wichtigen ersten Schritt dar, mögliche Einflüsse der heterogenen Studienstrukturen in der Lehramtsausbildung auf die Entwicklung der professionellen Kompetenz angehender Lehrkräfte zu identifizieren.

Prospektiv können die hier vorgestellten Ergebnisse dafür genutzt werden, Studiengänge anhand ihrer fachlichen und fachdidaktischen Anteile genauer zu klassifizieren und mögliche Schwächen in der Abdeckung der Inhalte zu identifizieren. Dies bedeutet insbesondere, dass Hochschulen zukünftig ihre fachdidaktische Ausbildung hinsichtlich der ländergemeinsamen Anforderungen (KMK 2019) neu strukturieren sollten. Durch die Darstellung der verschiedenen Möglichkeiten, die sich für das Physik-Lehramtsstudium ergeben, können Hochschulen ihre Studiengänge zukünftig evaluieren und den Einfluss auf den Wissenserwerb angehender Physiklehrkräfte einschätzen. So sollten Hochschulen die fachdidaktische Ausbildung in den ersten sechs Semestern stärker fokussieren und insbesondere relevante Inhalte explizit thematisieren, statt lediglich überblicksartig zu lehren. Zudem zeigt die vor- 
liegende Studie auch, dass die fachdidaktische Ausbildung von Physiklehrkräften in den ersten sechs Semestern weitestgehend losgelöst von den ländergemeinsamen Anforderungen (KMK 2019) verläuft, da vorgeschriebene Inhaltsbereiche kaum thematisiert werden. Die Bildungspolitik der Länder sowie die KMK sollten sich also zwei Fragen stellen: zum einen, welche fachdidaktischen Inhalte Physiklehrkräfte tatsächlich benötigen und zum anderen, wie sich diese Inhalte durch verbindliche und realisierbare Anforderungen bereits im ersten Teil der universitären Lehramtsausbildung implementieren lassen. Auf diese Weise können Studiengänge und nachfolgend auch die Professionalisierung angehender Physiklehrkräfte zukünftig weiter optimiert werden.

Funding Open Access funding enabled and organized by Projekt DEAL.

Open Access Dieser Artikel wird unter der Creative Commons Namensnennung 4.0 International Lizenz veröffentlicht, welche die Nutzung, Vervielfältigung, Bearbeitung, Verbreitung und Wiedergabe in jeglichem Medium und Format erlaubt, sofern Sie den/die ursprünglichen Autor(en) und die Quelle ordnungsgemäß nennen, einen Link zur Creative Commons Lizenz beifügen und angeben, ob Änderungen vorgenommen wurden.

Die in diesem Artikel enthaltenen Bilder und sonstiges Drittmaterial unterliegen ebenfalls der genannten Creative Commons Lizenz, sofern sich aus der Abbildungslegende nichts anderes ergibt. Sofern das betreffende Material nicht unter der genannten Creative Commons Lizenz steht und die betreffende Handlung nicht nach gesetzlichen Vorschriften erlaubt ist, ist für die oben aufgeführten Weiterverwendungen des Materials die Einwilligung des jeweiligen Rechteinhabers einzuholen.

Weitere Details zur Lizenz entnehmen Sie bitte der Lizenzinformation auf http://creativecommons.org/ licenses/by/4.0/deed.de.

\section{Literatur}

Bauer, J., Diercks, U., Retelsdorf, J., Kauper, T., Zimmermann, F., Köller, O., Möller, J., \& Prenzel, M. (2011). Spannungsfeld Polyvalenz in der Lehrerbildung. Zeitschrift für Erziehungswissenschaft, 14(4), 629-649. https://doi.org/10.1007/s11618-011-0239-7.

Bauer, J., Diercks, U., Rösler, L., Möller, J., \& Prenzel, M. (2012). Lehramtsstudium in Deutschland: Wie groß ist die strukturelle Vielfalt? Unterrichtswissenschaft, 40(2), 101-120.

Baumert, J., \& Kunter, M. (2006). Stichwort: Professionelle Kompetenz von Lehrkräften. Zeitschrift für Erziehungswissenschaft, 9(4), 469-520.

Baumert, J., \& Kunter, M. (2011). Das Kompetenzmodell von COACTIV. In M. Kunter, J. Baumert, W. Blum, U. Klusmann, S. Krauss \& M. Neubrand (Hrsg.), Professionelle Kompetenz von Lehrkräften: Ergebnisse des Forschungsprogramms COACTIV (S. 29-53). Münster: Waxmann.

Baumert, J., Kunter, M., Blum, W., Brunner, M., Voss, T., Jordan, A., Klusmann, U., Krauss, S., Neubrand, M., \& Tsai, Y.-M. (2010). Teachers' mathematical knowledge, cognitive activation in the classroom, and student progress. American Educational Research Journal, 47(1), 133-180. https://doi.org/10. 3102/0002831209345157.

Blömeke, S., Gustafsson, J.-E., \& Shavelson, R. J. (2015). Beyond dichotomies: competence viewed as a continuum. Zeitschrift für Psychologie, 223(1), 3-13. https://doi.org/10.1027/2151-2604/a000194.

Bond, T.G., \& Fox, C.M. (2007). Applying the Rasch model: fundamental measurement in the human sciences (2. Aufl.). New Jersey: Lawrence Erlbaum.

Borowski, A., Neuhaus, B. J., Tepner, O., Wirth, J., Fischer, H. E., Leutner, D., Sandmann, A., \& Sumfleth, E. (2010). Professionswissen von Lehrkräften in den Naturwissenschaften (ProwiN): Kurzdarstellung des BMBF-Projekts. Zeitschrift für Didaktik der Naturwissenschaften, 16, 341-349.

Brunner, M., Kunter, M., Krauss, S., Baumert, J., Blum, W., Dubberke, T., Jordan, A., Klusmann, U., Tsai, Y.-M., \& Neubrand, M. (2006). Welche Zusammenhänge bestehen zwischen dem fachspezifischen 
Professionswissen von Mathematiklehrkräften und ihrer Ausbildung sowie beruflichen Fortbildung? Zeitschrift für Erziehungswissenschaft, 9(4), 521-544. https://doi.org/10.1007/s11618-006-0166-1.

Carlson, J., \& Daehler, K. R. (2019). The refined consensus model of pedagogical content knowledge in science education. In A. Hume, R. Cooper \& A. Borowski (Hrsg.), Repositioning pedagogical content knowledge in teachers' knowledge for teaching science (S. 77-92). Singapore: Springer.

Deutsche Physikalische Gesellschaft (DPG) (2014). Zur fachlichen und fachdidaktischen Ausbildung für das Lehramt Physik. Bad Honnef: Deutsche Physikalische Gesellschaft.

Eurydice (2010). Fokus auf die Hochschulbildung in Europa 2010: Die Auswirkungen des Bologna-Prozesses. Brüssel: Education Audiovisual and Culture Executive Agency P9 Eurydice.

Fischer, H.E., Labudde, P., Neumann, K., \& Viiri, J. (Hrsg.). (2014). Quality of instruction in physics: comparing Finland, Switzerland and Germany (1. Aufl.). Münster: Waxmann. http://www.contentselect.com/index.php?id=bib_view\&ean=9783830980551.

Gess-Newsome, J. (2015). A model of teacher professional knowledge and skill including PCK: results of the thinking from the PCK summit. In A. Berry, P. J. Friedrichsen \& J. Loughran (Hrsg.), Teaching and learning in science series. Re-examining pedagogical content knowledge in science education (1. Aufl., S. 28-42). London: Routledge.

Gramzow, Y., Riese, J., \& Reinhold, P. (2013). Modellierung fachdidaktischen Wissens angehender Physiklehrkräfte. Zeitschrift für Didaktik der Naturwissenschaften, 19, 7-30.

Grossman, P.L. (1990). The making of a teacher: teacher knowledge and teacher education. New York: Teachers College.

Großschedl, J., Harms, U., Kleickmann, T., \& Glowinski, I. (2015). Preservice biology teachers' professional knowledge: structure and learning opportunities. Journal of Science Teacher Education, 26(3), 291-318. https://doi.org/10.1007/s10972-015-9423-6.

Hartig, J., \& Kühnbach, O. (2006). Schätzung von Veränderung mit „plausible values“ in mehrdimensionalen Rasch-Modellen. In I. A. Ittel \& H. Merkens (Hrsg.), Veränderungsmessung und Längsschnittstudien in der empirischen Erziehungswissenschaft (1. Aufl., S. 27-44). Wiesbaden: VS. https://doi. org/10.1007/978-3-531-90502-0_3.

Hartigan, J. A., \& Wong, M. A. (1979). Algorithm AS 136: a k-means clustering algorithm. Journal of the Royal Statistical Society. Series C (Applied Statistics), 28(1), 100-108.

Hascher, T. (2011). Forschung zur Wirksamkeit der Lehrerbildung. In E. Terhart, H. Bennewitz \& M. Rothland (Hrsg.), Handbuch der Forschung zum Lehrerberuf (S. 418-440). Münster: Waxmann.

Kassambara, A. (2017). Practical guide to cluster analysis in R: unsupervised machine learning (1. Aufl.). STHDA.

Kassambara, A., \& Mundt, F. (2017). factoextra: extract and visualize the results of multivariate data analyses: R package version 1.0.5. https://CRAN.R-project.org/package=factoextra. Zugegriffen: 07. Sept. 2020

Kaufman, L., \& Rousseeuw, P. J. (1990). Finding groups in data: an introduction to cluster analysis. Hoboken: Wiley.

Keller, A. (2010). Gleichermaßen hochwertig und einheitlich lang: Gewerkschaftliche Anforderungen an die Reform der Lehrerbildung im Bologna-Prozess. Erziehungswissenschaft, 21(40), 99-107.

Keuffer, J. (2010). Reform der Lehrerbildung und kein Ende? Eine Standortbestimmung. Erziehungswissenschaft, 21(40), 51-67.

Kind, V. (2009). Pedagogical content knowledge in science education: perspectives and potential for progress. Studies in Science Education, 45(2), 169-204. https://doi.org/10.1080/03057260903142285.

Kind, V., \& Chan, K. K.H. (2019). Resolving the amalgam: connecting pedagogical content knowledge, content knowledge and pedagogical knowledge. International Journal of Science Education. https:// doi.org/10.1080/09500693.2019.1584931.

Kirschner, S. (2013). Modellierung und Analyse des Professionswissens von Physiklehrkräften (Studien zum Physik- und Chemielernen, Bd. 161). Berlin: Logos.

Kirschner, S., Borowski, A., Fischer, H. E., Gess-Newsome, J., \& von Aufschnaiter, C. (2016). Developing and evaluating a paper-and-pencil test to assess components of physics teachers' pedagogical content knowledge. International Journal of Science Education, 38(8), 1343-1372.

Kirschner, S., Sczudlek, M., Tepner, O., Borowski, A., Fischer, H.E., Lenske, G., Leutner, D., Neuhaus, B. J., Sumfleth, E., Thillmann, H., \& Wirth, J. (2017). Professionswissen in den Naturwissenschaften (ProwiN). In C. Gräsel \& K. Trempler (Hrsg.), Entwicklung von Professionalität pädagogischen Personals: Interdisziplinäre Betrachtungen, Befunde und Perspektiven (S. 113-130). Wiesbaden: Springer VS. https://doi.org/10.1007/978-3-658-07274-2_7. 
Kleickmann, T., \& Anders, Y. (2011). Lernen an der Universität. In M. Kunter, J. Baumert, W. Blum, U. Klusmann, S. Krauss \& M. Neubrand (Hrsg.), Professionelle Kompetenz von Lehrkräften: Ergebnisse des Forschungsprogramms COACTIV (S. 305-315). Münster: Waxmann.

Kleickmann, T., Richter, D., Kunter, M., Elsner, J., Besser, M., Krauss, S., \& Baumert, J. (2013). Teachers' content knowledge and pedagogical content knowledge. Journal of Teacher Education, 64(1), 90-106. https://doi.org/10.1177/0022487112460398.

Kleickmann, T., Großschedl, J., Harms, U., Heinze, A., Herzog, S., Hohenstein, F., Köller, O., Kröger, J., Lindmeier, A., Loch, C., Mahler, D., Möller, J., Neumann, K., Parchmann, I., Steffensky, M., Taskin, V., \& Zimmermann, F. (2014). Professionswissen von Lehramtsstudierenden der mathematisch-naturwissenschaftlichen Fächer - Testentwicklung im Rahmen des Projekts KiL. Unterrichtswissenschaft, 42(3), 280-288.

KMK (2005) = Kultusministerkonferenz der Bundesrepublik Deutschland. (2005). Eckpunkte für die gegenseitige Anerkennung von Bachelor- und Masterabschlüssen in Studiengängen, mit denen die Bildungsvoraussetzungen für ein Lehramt vermittelt werden.

KMK (2019) = Kultusministerkonferenz der Bundesrepublik Deutschland. (2019). Ländergemeinsame inhaltliche Anforderungen für die Fachwissenschaften und Fachdidaktiken in der Lehrerbildung (Beschluss der Kultusministerkonferenz der Bundesrepublik Deutschland vom 16.10.2008 i.d. F. vom 16.05.2019).

Korneck, F., Krüger, M., \& Szogs, M. (2017). Professionswissen, Lehrerüberzeugungen und Unterrichtsqualität angehender Physiklehrkräfte unterschiedlicher Schulformen. In E. Sumfleth \& H. Fischler (Hrsg.), Professionelle Kompetenzen von Lehrkräften der Chemie und Physik.: Studien zum Physikund Chemielernen (S. 113-133). Berlin: Logos.

Krauss, S. (2011). Das Experten-Paradigma in der Forschung zum Lehrerberuf. In E. Terhart, H. Bennewitz \& M. Rothland (Hrsg.), Handbuch der Forschung zum Lehrerberuf(S. 171-191). Münster: Waxmann.

Krauss, S., Brunner, M., Kunter, M., Baumert, J., Blum, W., Neubrand, M., \& Jordan, A. (2008). Pedagogical content knowledge and content knowledge of secondary mathematics teachers. Journal of Educational Psychology, 100(3), 716-725. https://doi.org/10.1037/0022-0663.100.3.716.

Kröger, J. (2019). Struktur und Entwicklung des Professionswissens angehender Physiklehrkräfte. Dissertation. https://nbn-resolving.org/urn:nbn:de:gbv:8-diss-251193. Zugegriffen: 07. Sept. 2020.

Kulgemeyer, C., \& Riese, J. (2018). From professional knowledge to professional performance: the impact of CK and PCK on teaching quality in explaining situations. Journal of Research in Science Teaching, 55(10), 1393-1418. https://doi.org/10.1002/tea.21457.

Kunina-Habenicht, O., Schulze-Stocker, F., Kunter, M., Baumert, J., Leutner, D., Förster, D., Lohse-Bossenz, H., \& Terhart, E. (2013). Die Bedeutung der Lerngelegenheiten im Lehramtsstudium und deren individuelle Nutzung für den Aufbau des bildungswissenschaftlichen Wissens. Zeitschrift für Pädagogik, 59(1), 1-23. https://doi.org/10.7767/boehlau.9783205789703.7.

Liepertz, S., \& Borowski, A. (2018). Testing the Consensus Model: relationships among physics teachers' professional knowledge, interconnectedness of content structure and student achievement. International Journal of Science Education, 37(3), 1-21. https://doi.org/10.1080/09500693.2018.1478165.

MacQueen, J. (1967). Some methods for classification and analysis of multivariate observations. In L. M. Le Cam \& J. Neyman (Hrsg.), Proceedings of the Fifth Berkeley Symposium on Mathematical Statistics and Probability (Bd. 1, S. 281-297). Berkley: University of California Press.

Magnusson, S., Krajcik, J., \& Borko, H. (1999). Nature, sources and development of pedagogical content knowledge for science teaching. In J. Gess-Newsome \& N. G. Lederman (Hrsg.), Examining pedagogical content knowledge: the construct and its implications for science education (S. 95-132). Dodrecht: Springer.

Neumann, K., Kind, V., \& Harms, U. (2018). Probing the amalgam: the relationship between science teachers' content, pedagogical and pedagogical content knowledge. International Journal of Science Education, 16(11), 1-15. https://doi.org/10.1080/09500693.2018.1497217.

Park, S., \& Oliver, J. S. (2008). Revisiting the conceptualisation of pedagogical content knowledge (PCK): PCK as a conceptual tool to understand teachers as professionals. Research in Science Education, 38(3), 261-284. https://doi.org/10.1007/s11165-007-9049-6.

Radhoff, M., \& Ruberg, C. (2016). Die Lehramtsausbildung. In H.-C. Koller, H. Faulstich-Wieland, H. Weishaupt \& I. Züchner (Hrsg.), Schriften der Deutschen Gesellschaft für Erziehungswissenschaft (DGfE). Datenreport Erziehungswissenschaft 2016 (S. 41-69). Opladen: Barbara Budrich.

Riese, J. (2009). Professionelles Wissen und professionelle Handlungskompetenz von (angehenden) Physiklehrkräften (Studien zum Physik- und Chemielernen, Bd. 97). Berlin: Logos. 
Riese, J., \& Reinhold, P. (2010). Empirische Erkenntnisse zur Struktur professioneller Handlungskompetenz von angehenden Physiklehrkräften. Zeitschrift für Didaktik der Naturwissenschaften, 16, $167-187$.

Riese, J., \& Reinhold, P. (2012). Die professionelle Kompetenz angehender Physiklehrkräfte in verschiedenen Ausbildungsformen: Empirische Hinweise für eine Verbesserung des Lehramtsstudiums. Zeitschrift für Erziehungswissenschaft, 15(1), 111-143. https://doi.org/10.1007/s11618-012-0259-y.

Riese, J., Kulgemeyer, C., Zander, S., Borowski, A., Fischer, E. H., Gramzow, Y., Reinhold, P., Schecker, H., \& Tomczyszyn, E. (2015). Modellierung und Messung des Professionswissens in der Lehramtsausbildung Physik. Zeitschrift für Pädagogik, 61(61), 55-79.

Riese, J., Gramzow, Y., \& Reinhold, P. (2017). Die Messung fachdidaktischen Wissens bei Anfängern und Fortgeschrittenen im Lehramtsstudiengang Physik. Zeitschrift für Didaktik der Naturwissenschaften, 23(1), 99-112. https://doi.org/10.1007/s40573-017-0059-2.

Robitzsch, A., Kiefer, T., \& Wu, M. (2019). TAM: Test Analysis Modules. R package version 3.2-24. https://CRAN.R-project.org/package=TAM. Zugegriffen: 07. Sept. 2020

Rousseeuw, P. J. (1987). Silhouettes: a graphical aid to the interpretation and validation of cluster analysis. Journal of Computational and Applied Mathematics, 20, 53-65.

Schiering, D., Sorge, S., Petersen, S., \& Neumann, K. (2019). Konstruktion eines qualitativen Niveaumodells im fachdidaktischen Wissen von angehenden Physiklehrkräften. Zeitschrift für Didaktik der Naturwissenschaften, 25(1), 211-229. https://doi.org/10.1007/s40573-019-00100-y.

Schmidt, W.H., Tatto, M.T., Bankov, K., Blömeke, S., Cedillo, T., Cogan, L., Shin, I.H., Houang, R., Hsieh, F. J., Paine, L., Santillan, M., \& Schwille, J. (2007). The preparation gap: teacher education for middle school mathematics in six countries (MT21 Report).

Schödl, A. (2017). FALKO-Physik - Fachspezifische Lehrerkompetenzen im Fach Physik: Entwicklung und Validierung eines Testinstruments zur Erfassung des fachspezifischen Professionswissens von Physiklehrkräften (Studien zum Physik- und Chemielernen, Bd. 236. Dissertation). Berlin: Logos Verlag.

Schröder, J., Riese, J., Vogelsang, C., Borowski, A., Buschhüter, D., Enkrott, P., Kempin, M., Kulgemeyer, C., Reinhold, P., \& Schecker, H. (2020). Die Messung der Fähigkeit zur Unterrichtsplanung im Fach Physik mit Hilfe eines standardisierten Performanztests. Zeitschrift für Didaktik der Naturwissenschaften. https://doi.org/10.1007/s40573-020-00115-w.

Sharafi, A. (2013). Knowledge Discovery in Databases: Eine Analyse des Änderungsmanagements in der Produktentwicklung. München: Springer.

Shavelson, R. J., Ruiz-Primo, M. A., \& Wiley, E. W. (2005). Windows into the mind. Higher Education, 49, 413-430. https://doi.org/10.1007/s10734-004-9448-9.

Shulman, L.S. (1986). Those who understand: knowledge growth in teaching. Educational Researcher, 15(4), 4-14.

Shulman, L.S. (1987). Knowledge and teaching: foundations of the new reform. Harvard Educational Review, 57(1), 1-22.

Sorge, S., Kröger, J., Petersen, S., \& Neumann, K. (2019). Structure and development of pre-service physics teachers' professional knowledge. International Journal of Science Education, 41(7), 862-889. https://doi.org/10.1080/09500693.2017.1346326.

Straube, P. (2016). Modellierung und Erfassung von Kompetenzen naturwissenschaftlicher Erkenntnisgewinnung bei (Lehramts-)Studierenden im Fach Physik (Studien zum Physik- und Chemielernen, Bd. 209. Dissertation). Berlin: Logos.

Tachtsoglou, S., \& König, J. (2017). Der Einfluss universitärer Lerngelegenheiten auf das pädagogische Wissen von Lehramtsstudierenden. Zeitschrift für Bildungsforschung, 7(3), 291-310. https://doi.org/ 10.1007/s35834-017-0199-y.

Tepner, O., Borowski, A., Dollny, S., Fischer, H.E., Jüttner, M., Kirschner, S., Leutner, D., Neuhaus, B. J., Sandmann, A., Sumfleth, E., Thillmann, H., \& Wirth, J. (2012). Modell zur Entwicklung von Testitems zur Erfassung des Professionswissens von Lehrkräften in den Naturwissenschaften. Zeitschrift für Didaktik der Naturwissenschaften, 19(1), 7-28.

Terhart, E. (2008). Die Lehrerbildung. In K. S. Cortina, J. Baumert, A. Leschinsky, K. U. Mayer \& L. Trommer (Hrsg.), Das Bildungswesen in der Bundesrepublik Deutschland. Strukturen und Entwicklungen im Überblick: der neue Bericht des Max-Planck-Instituts für Bildungsforschung (S. 745-772). Reinbek: Rowohlt.

Terhart, E. (2012). Wie wirkt Lehrerbildung? Forschungsprobleme und Gestaltungsfragen. Zeitschrift für Bildungsforschung, 2(1), 3-21. https://doi.org/10.1007/s35834-012-0027-3. 
Trendtel, M., Pham, G., \& Yanagida, T. (2016). Skalierung und Linking. In S. Breit \& C. Schreiner (Hrsg.), Large-Scale Assessment mit R: Methodische Grundlagen der österreichischen Bildungsstandardüberprüfung (S. 185-224). Wien: facultas.

Vogelsang, C., Borowski, A., Kulgemeyer, C., Riese, J., Buschhüter, D., Enkrott, P., Kempin, M., Reinhold, P., Schecker, H., \& Schröder, J. (2020). Development of prospective physics teachers' professional knowledge and skills during a one-semester school internship. In O. Zlatkin-Troitschanskaia, H. A. Pant, M. Toepper \& C. Lautenbach (Hrsg.), Student learning in German higher education: innovative measurement approaches and research results (S. 105-123). Wiesbaden: Springer VS.

Watson, C., Seifert, A., \& Schaper, N. (2018). Die Nutzung institutioneller Lerngelegenheiten und die Entwicklung bildungswissenschaftlichen Wissens angehender Lehrkräfte. Zeitschrift für Erziehungswissenschaft, 21(3), 565-588. https://doi.org/10.1007/s11618-017-0794-7.

Weiler, H. N. (2007). Neue Wege in der Lehrerausbildung: Staatliche und universitäre Verantwortlichkeiten. In D. Lemmermöhle, M. Rothgangel, S. Bögeholz, M. Hasselhorn \& R. Watermann (Hrsg.), Professionell lehren, erfolgreich lernen (S. 23-50). Münster: Waxmann.

Woitkowski, D. (2020). Tracing physics content knowledge gains using content complexity levels. International Journal of Science Education, 17(2), 1-24. https://doi.org/10.1080/09500693.2020.1772520. 